\title{
A Novel Coverage Improved Deployment Strategy for Wireless Sensor Network
}

Sathees Lingam Paulswamy ( $\nabla$ satheeslingampaulswamy@gmail.com )

Priyadarshini College of Engineering

A.Andrew Roobert

Francis Xavier Engineering College

K. Hariharan

Thiagarajar College of Engineering

\section{Research Article}

Keywords: Sensor Network, Node Deployment, Area Coverage, Disc Shape Deployment

Posted Date: March 16th, 2021

DOI: https://doi.org/10.21203/rs.3.rs-291535/v1

License: (1) This work is licensed under a Creative Commons Attribution 4.0 International License. Read Full License

Version of Record: A version of this preprint was published at Wireless Personal Communications on December 1st, 2021. See the published version at https://doi.org/10.1007/s11277-021-09387-y. 


\title{
A novel coverage improved deployment strategy for wireless sensor network
}

\author{
Sathees Lingam Paulswamy ${ }^{1}$, A.Andrew Roobert ${ }^{2}$, K.Hariharan ${ }^{3}$ \\ Department of Electronics and Communication Engineering, \\ ${ }^{1}$ Priyadarshini College of Engineering, Nellore, Andhra Pradesh - 524004, India. \\ ${ }^{2}$ Francis Xavier Engineering College, Tirunelveli, Tamilnadu - 627003, India \\ ${ }^{3}$ Thiagarajar College of Engineering, Madurai, Tamilnadu - 625015, India \\ *Correspondence to: Sathees Lingam Paulswamy, \\ Email: satheeslingampaulswamy@gmail.com
}

\section{Summary}

Coverage of the bounded region gets importance in Wireless Sensor Network (WSN). Area coverage is based on effective surface coverage with a minimum number of sensor nodes. Most of the researchers contemplate the coverage region of interest as a square and manifest the radio ranges as a circle. The area of a circle is much higher than the area of a square because of the perimeter. To utilize the advantage of the circle, the coverage region of interest is presumed as a circle for sensor node deployment. This paper proposes a novel coverage improved disc shape deployment strategy. Comparative analysis has been observed between circle and square regions of interest based on the cumulative number of sensor nodes required to cover the entire region. A new strategy named as disc shape deployment strategy is also proposed. Traditional hexagon and strip-based deployment strategies are compared with the disc shape deployment strategy. The simulation result shows that the circle shape coverage region of interest extremely reduces the required number of sensor nodes. The proposed deployment strategy provides desirable coverage, and it requires few more sensor nodes than hexagon shape deployment strategy.

\section{KEYWORDS}

Sensor Network; Node Deployment; Area Coverage; Disc Shape Deployment. 


\section{INTRODUCTION}

In an environment monitoring system, WSN is used to gather and transmit the information from the environment to Base Station (BS). Two dissimilar sensor node deployment strategies namely random and deterministic are used. Each sensor node comprises a sensing unit, processing unit and communication unit. The sensing unit and processing unit ingest much less energy when compared to the communication unit [1]. Communication takes place by single-hop and multi-hop in WSN, where single-hop communication is mostly concentrated on centralized deployment, and the multi-hop communication is concentrated on distributive deployment. WSN can be either homogenous or heterogeneous based on deployment strategy. Each node has the same energy level, sensing range, communication range and processing capabilities in a homogenous sensor network. On the other hand, each node has dissimilar sensing range, energy level, communication range and processing capabilities in a heterogeneous sensor network.

Communication in WSN is highly relying on the characteristics of the antenna. In an Omnidirectional antenna, the formation of the communication range and sensing range has been considered as a circle. As per various researches, the Omnidirectional antenna is distinctly suitable for WSN, because each node should be able to communicate with entire neighbor nodes, which are placed within the communication range. According to distinct research analyzes in WSN, the neighbor node is essential to place within the communication range. If the sensor nodes are not properly placed (beyond communication range), the whole network will be collapsed, which originates coverage hole on the field.

Nowadays, the deployment is one of the rudimentary issues in WSN. Node deployment can be done in a random manner as well as a deterministic manner. Sensor node hurls from a helicopter or air vehicle for military surveillance applications is one of the examples for random deployment. In the random deployment, each node doesn't know its location, and some of the nodes may not be dropped properly within the coverage area. It requires mobility to reach the coverage area, but it may not be easy to 
determine the exact location of the sensor node. In deterministic deployment, maximum coverage in the region can be achieved effortlessly, and it can easily determine the location of the sensor node. The coverage has been assorted based on the range of radio signals and the maximum allowable distance [2]. Two sensors can directly exchange information when the distance is lower than the communication range ' $\mathrm{R}_{\mathrm{C}}$ ' [3].

Neighbor nodes should be deployed within the communication range for getting connectivity, where the Communication Range $\left(R_{C}\right)$ will be twice the Sensing Range $\left(R_{S}\right)$, but the communication range is tremendously assorted based on the energy availability in a battery. The detection probability is based on the distance variation between neighbor nodes [4]. GPS (Global Positioning System) device has been affixed with the sensor node for constructing the system into a location-aware one.

Mobile sensor node's mobility ingests much energy while employing a GPS device. High power consumption is not complementary for wireless sensor networks [5]. Otherwise, the location of the sensor node determined based on the presumption of origin node. It is not an appropriate global assignment for coordinate within the coverage region at WSN [6].

The location of the origin and router has not been mentioned in the deployment of the sensor node. At the same time, due to the unnecessary assignment of the router node, which generates redundancy in the sensor network [7]. Equilateral triangle tessellation has been used for deployment of sensor node, where GA and GRG algorithms are used only for route discovery towards the sink. There was no genuine analysis given for area coverage (bounded or unbounded). Equilateral tessellation initiates coverage hole nearby the boundary at bounded area coverage [8]. Strip based deployment strategy produces coverage hole nearby boundary of the coverage region. Some supplementary nodes should be affixed to improve the coverage $[9,10]$.

Selective insertion of the supplementary node is not vastly desirable within the coverage region, because sometimes it produces unwanted redundancy [10]. Hexagon honey comp structure is the optimal pattern 
for the unbounded area. Adopting the honey comp structure into the bounded region coverage requires some secondary sensor nodes, which are not recommended [11, 12, 13]. The deployment is highly related to the selection of the shape of the coverage region of interest and deployment patterns. Predominantly the researchers presume that the coverage region of interest as square, and they opt the equilateral triangle tessellation as a successful deployment pattern. Both are not pledged competent coverage in the bounded region $[14,15,16,17]$.

They have faced the problem of coverage hole, due to antithetical in shape between communications range and coverage region of interest. To solve such an antithetical problem of square shape region, the structure of the coverage region of interest is taken as circular in the proposed strategy. Moreover, in this paper, comparative analysis has been done between various deployments strategies and our proposed deployment strategy.

This paper is organized as follows. Strip based deployment strategy in square shape region is discussed in Section 2. Hexagon shape deployment strategy is detailed in Section 3. Probabilistic model for disc shape deployment strategy is elaborated in Section 4. The conclusion is given in Section 5.

\section{STRIP BASED DEPLOYMENT STRATEGY IN SQUARE SHAPE REGION}

A good deployment should appraise both coverage and connectivity. Here, deterministic deployment has been used. It is distinctly preferable for many of the applications like agriculture, area surveillance, etc. The researchers are predominantly intense on the square shape $(R \times R)$ for the coverage region of interest. The communication range $\left(\mathrm{R}_{\mathrm{C}}\right)$ could be twice the sensing range $\left(\mathrm{R}_{\mathrm{S}}\right)$, which means ' $R c=2 \times$ $R s^{\prime}[3,18]$. A sensing model and communication model instigates based on the Omnidirectional antenna. 


\subsection{PROBABILISTIC SENSING MODEL}

Every sensor node has a disc-shaped sensing range with equal radius ' $\mathrm{R}_{\mathrm{S}}$ '. The probability of coverage of point ' $a$ ' is one when the distance between ' $a$ ' and origin is less than ' $R_{S}$ ', otherwise the probability of coverage will be zero. In Figure 1, the probability to cover a point 'a' is given by:

$$
\text { Prob. }(\text { coverage }(a))= \begin{cases}1, & d(\text { node }, a) \leq R s \\ 0, \text { otherwise }\end{cases}
$$

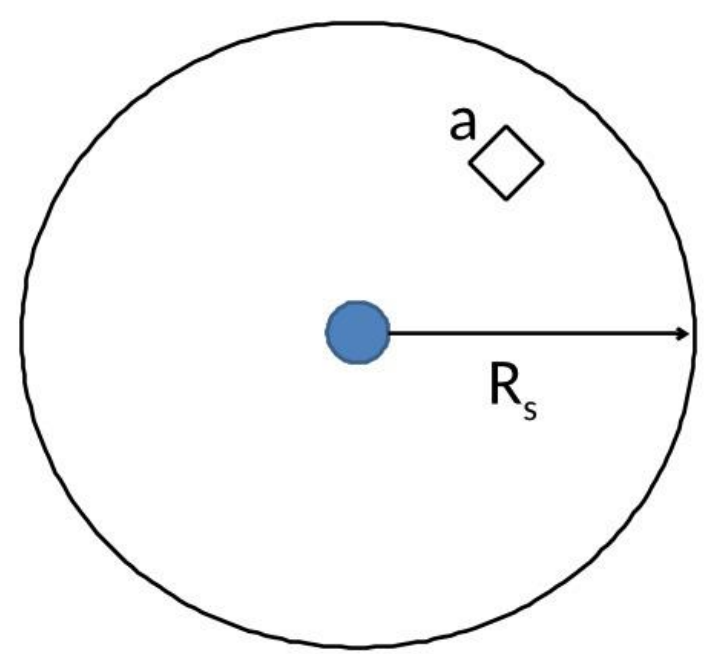

FIGURE 1 Geometry for sensing model

When the neighbor nodes are evenly separated by a distance of ' $R c \geq(\sqrt{3}) \times R s$ ', the connectivity is automatically guaranteed [19].

\subsection{PROBABILISTIC COMMUNICATION MODEL}

As shown in Figure 2, the neighbor node should be deployed within the Communication Range $\left(\mathrm{R}_{\mathrm{C}}\right)$ for getting connectivity. Building the communication between a pair of sensor nodes is a critical task. Traditionally the communication range is determined with the help of Received Signal Strength Indicator (RSSI). 
Distance between nodes is playing a vital role in this communication model. If a pair of node ' $U$ ' and ' $V$ ' has the distance, $d(U, V) \leq R$, they can directly communicate with each other [2]. The coordinate points of two nodes are $\mathrm{U}_{\mathrm{X}}, \mathrm{U}_{\mathrm{Y}}$ and $\mathrm{V}_{\mathrm{X}}, \mathrm{V}_{\mathrm{Y}}$. The distance between two nodes is written as,

$$
d(U, V)=\sqrt{(U x-V x)^{2}+(U y-V y)^{2}}
$$

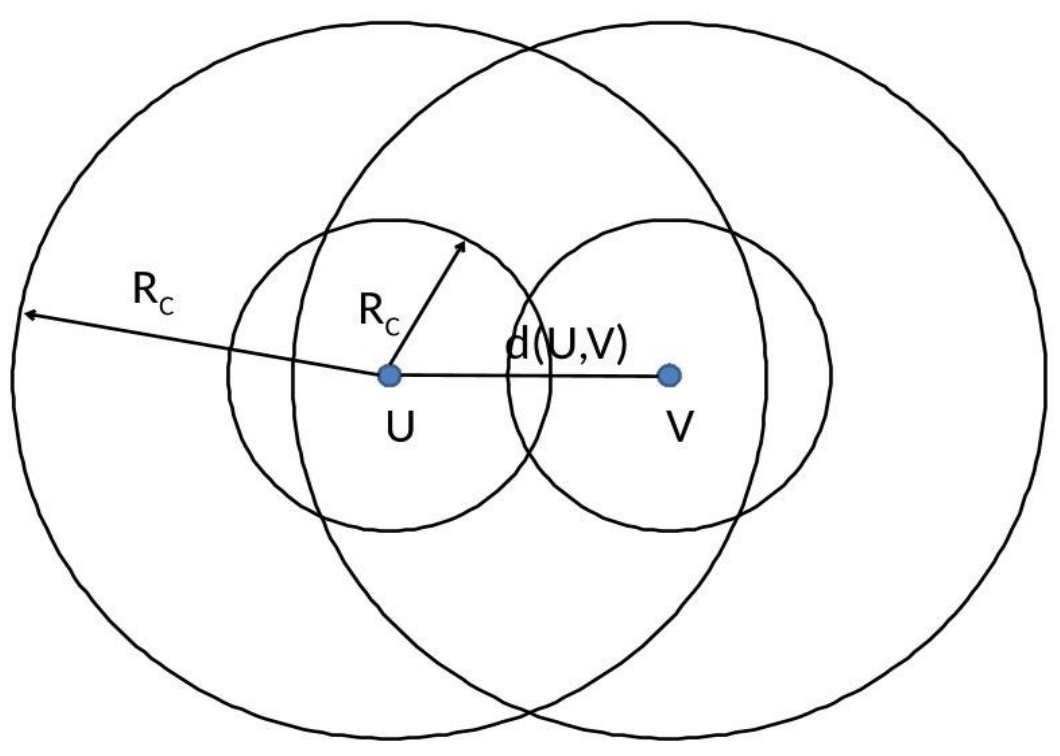

FIGURE 2 Geometry for a communication model

Where, $d(U, V)=$ distance between node ' $\mathrm{U}$ ' and node ' $\mathrm{V}$ '

In connectivity perspective, if neighbor nodes are evenly separated by a distance of $R c \leq(\sqrt{3}) \times R s$, the coverage will be granted. Probability of the communication between $\mathrm{U}$ and $\mathrm{V}$ is:

$$
\text { Prob. }(\text { connectivity }(a))=\left\{\begin{array}{c}
1, d(U, V) \leq R c \\
0, \text { otherwise }
\end{array}\right.
$$

\subsubsection{Geometry for Strip Based Deployment}

The coverage region of interest is considered as square shape ( $\mathrm{x} R$ ) when the strip based deployment strategy $[19,9,10,15]$ is instigated. The deployment of the first node initiates from the top left corner of the square. Remaining nodes are deployed in different rows and columns within the region under the 
condition of ' $R c=(\sqrt{3}) \times R s$ ' as depicted in Figure 3. This condition will grant preferable coverage and connectivity [19].

As per the strip-based deployment strategy, each node is evenly separated with the distance of $(\sqrt{3}) \times R s$ (horizontal). Every shift in the row (vertical) is calculated with the distance ' $((\sqrt{3}) \times R s) / 2$ ' along with horizontal deflection $c / 2\left(\frac{(\sqrt{3}) \times R s}{2}(\right.$ vertically $)+\frac{R c}{2}($ horizontally $\left.)\right)$. For example, sensor node deployment is shifted from the first row to the second row when the beginning node is deployed with the merged distance of $\frac{(\sqrt{3}) \times R s}{2}\left(\right.$ vertically) and $\frac{R c}{2}$ (horizontally). The remaining nodes are evenly separated with a distance of $(\sqrt{3}) \times R s$ as shown in the second row of Figure 3. This process will replicate until it covers the entire area of the square shaped coverage region.

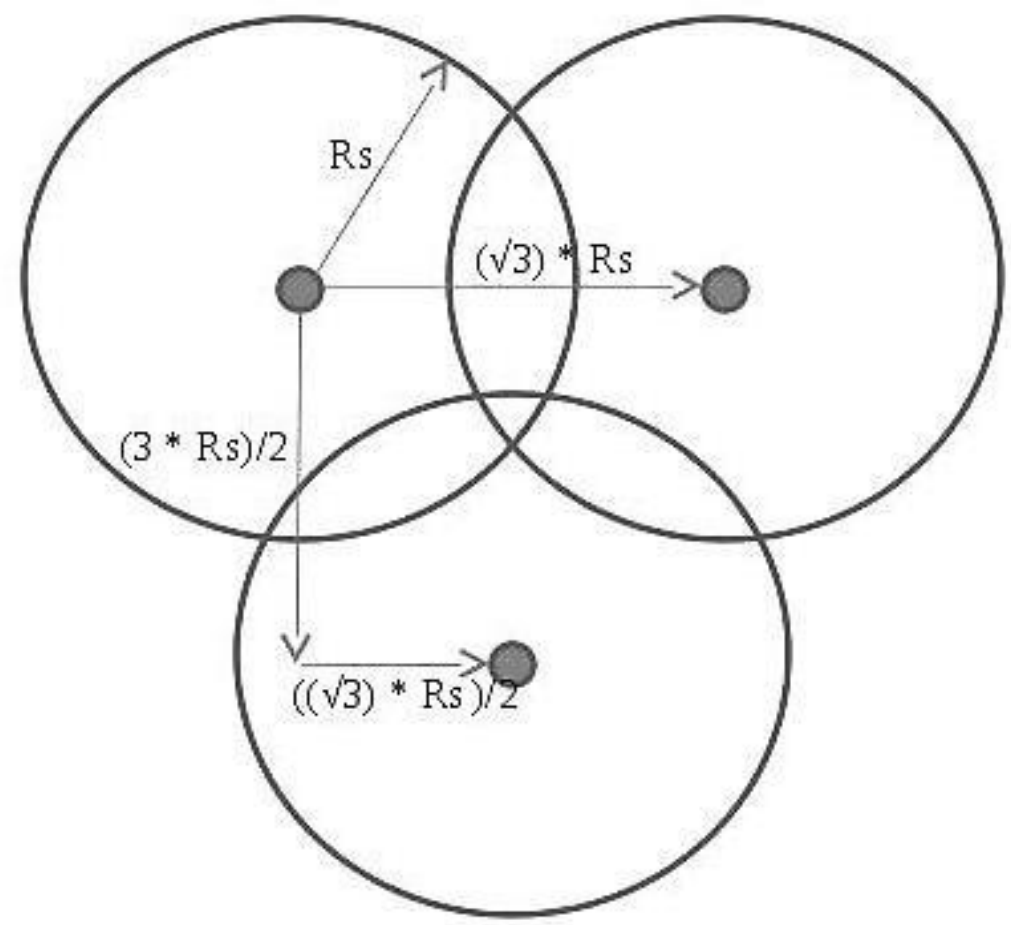

FIGURE 3 Geometry for strip-based deployment under the condition of $R c=(\sqrt{3}) \times R s$

Sensor node deployment sustained until it satisfies the condition ' $X \leq R$ (horizontally)' in each row. If the deployment of the sensor node in a new row exceeds the condition ' $X \leq R$ ', instantly node 
deployment will be shifted to the next row. Such vertical shift (downward) goes until it satisfies the condition ' $Y \geq 0$ '. When the node deployment exceeds the condition ' $Y \geq 0$ ', it stops the deployment. Strip based deployment predominantly depends on the boundary of coverage region of interest. Algorithm for strip-based deployment strategy in square shape coverage region is given below:

\section{Algorithm for Strip based deployment}

Step 1: Start

Step 2: Presume ' $\alpha=(\sqrt{3}) \times R s ; \beta=(3 \times R s) / 2 ; A=R \times R$ (square); $\mathrm{n}=1,2,3, \ldots, \infty$ '.

Step 3: Presume, the boundary of ' $X$ 'axis is $0-R$ (left to right) and the boundary of ' $Y$ ' axis is $0-$ $R$ (bottom to top).

Step 3: Place the reference node at $(\alpha, R-\alpha)$

Step 4: Deployment of remaining nodes (row, column)

Repeat Forward:

$$
\text { if }(X \leq R)
$$

Place the node with regular interval ' $\mathrm{na}$ ' distances from reference.

else

Shift down ' $\beta$ ' distance (vertically) and marginally move ' $R c / 2$ ' distance horizontally to deploy the first node of the another row

\section{Reverse:}

if $(X \geq 0)$

Deploy the sensor nodes reversely with regular interval ' $(-n \times \alpha)$ ' from the initial node of the second row.

else

Shift down ' $\beta$ ' distance (vertically) and marginally move ' $R c / 2$ ' distance (horizontally) to deploy the first node of the another row

$$
\text { if }(Y \geq 0)
$$

go Repeat

else 


\section{Step 5: Stop}

Based on the given geometry in Figure 3 and strip-based deployment algorithm, simulation has been done with the help of the Matlab 2014a version. The simulation results shown in Figure 4 and Figure 5 manifest that the extensive inequality in the total number of sensor nodes necessary to cover the entire area $(R \times R)$ in the square shape coverage region.

For example, if the square shape has the geometry of ' $10 \times 10$ ' units, it will require less number of nodes to cover the entire area as in Figure 4. If a square area of ' $20 \times 20$ ' units is used, it will require a massive number of nodes to cover the entire area as shown in Figure 5. Furthermore, the required number of nodes increases with the increment of geometry in the square shape coverage region.

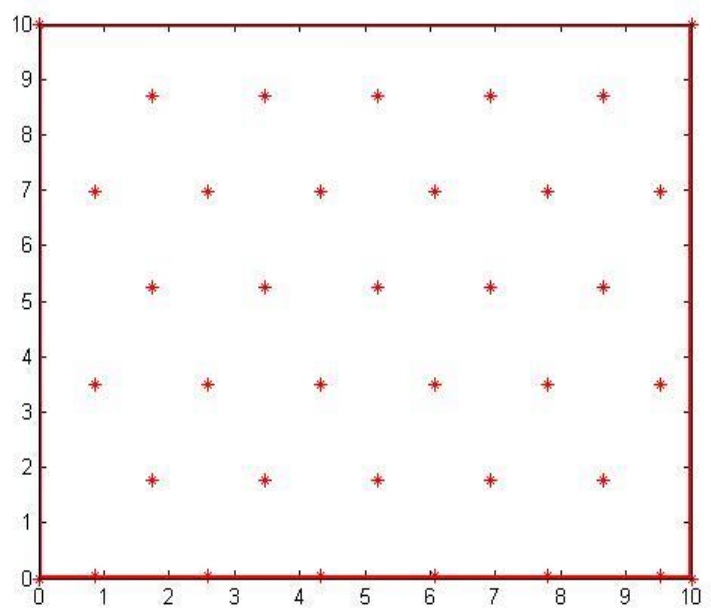

(a)

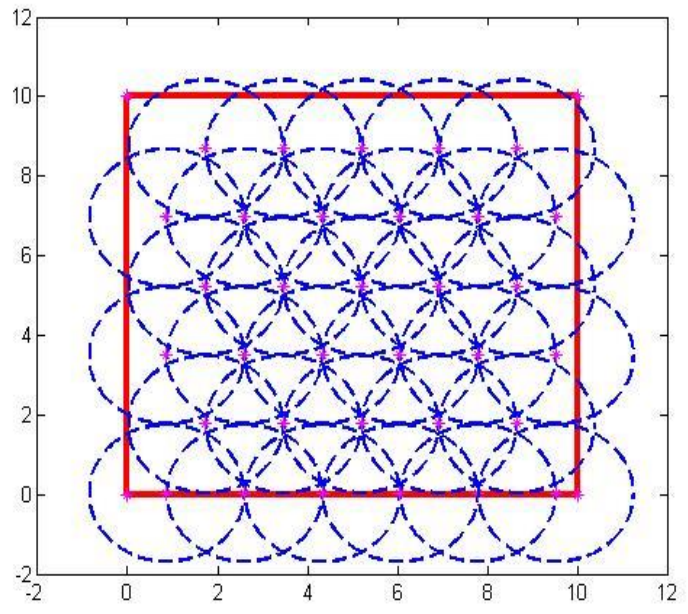

(b)

FIGURE 4 Square shape region's strip-based deployment for the geometry of $10 \times 10$ units. (a) Strip based deployment without sensing disc (b) Strip based deployment with sensing disc. 


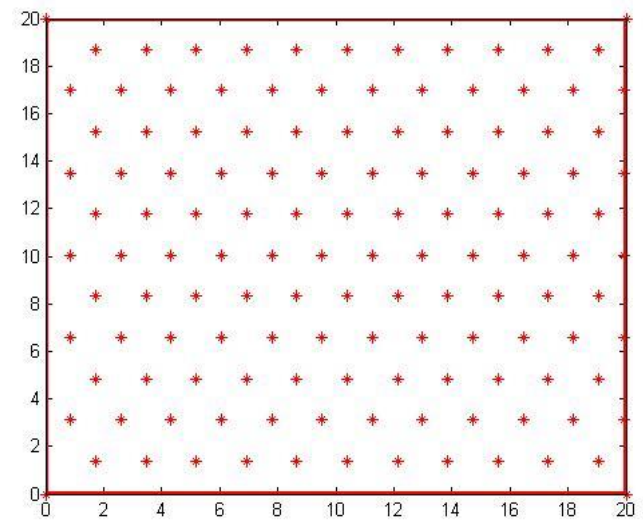

(a)

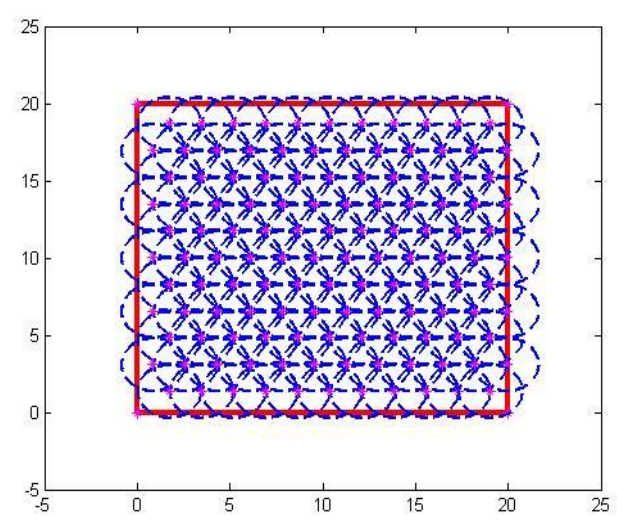

(b)

FIGURE 5 Square shape region's strip-based deployment for the geometry of $20 \times 20$ units. (a) Strip based deployment without sensing disc (b) Strip based deployment with sensing disc.

As per given geometry, there are plenty of coverage holes produced in Figure 6. Finding the uncovered area and deploying supplementary nodes at the specified location are extremely critical and ineffective tasks. Moreover, it is difficult to add the supplementary sensor nodes to cover the uncovered area.

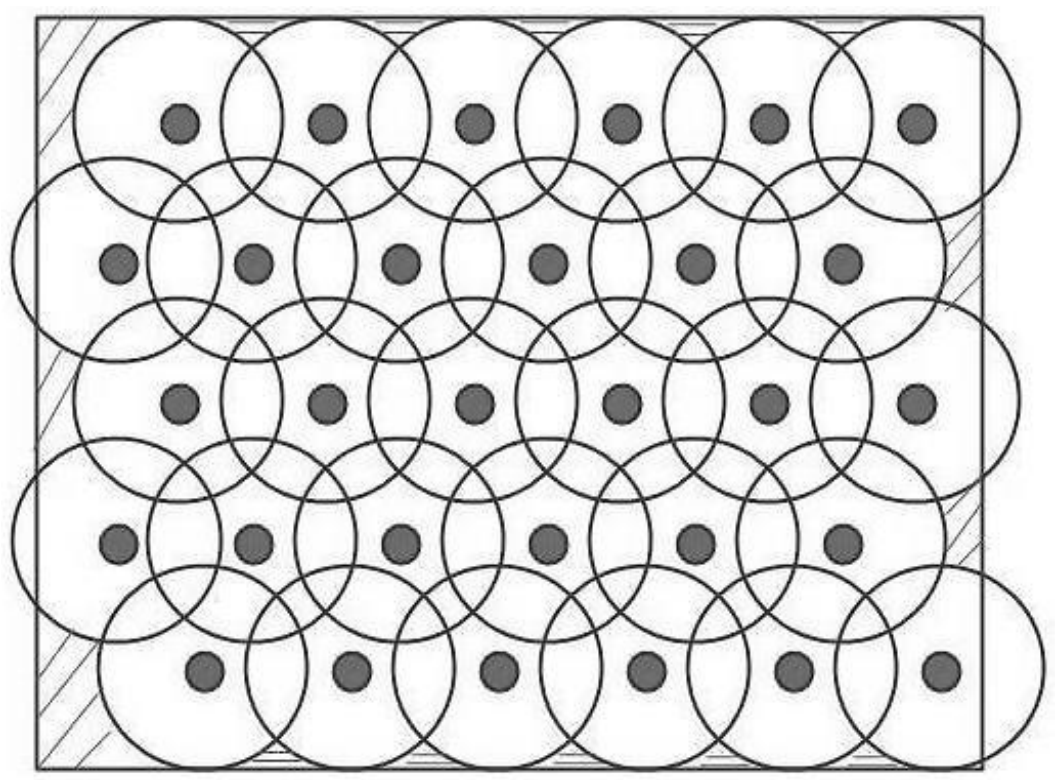

FIGURE 6 Coverage hole formation in the strip-based deployment

As per simulation, the coverage holes are produced by mismatching between the shape of the coverage region of interest and the shape of the communication range. The selection of coverage region of interest should be matched with the shape of the communication range to avoid the mismatch problem. Since the 
perimeter of circle is much higher than the perimeter of the square, the area of the circle is also much higher than the area of square [20]. The area equalization is salient contemplation between square shape coverage region of interest and circular shape coverage region of interest. Mathematical proof is given by:

$$
\begin{aligned}
& a^{2}=\pi r^{2} \\
& a=r \sqrt{\pi} \\
& r=a / \sqrt{\pi}
\end{aligned}
$$

To utilize the supremacy of circle, the presumption of coverage region of interest is a circle. Sensor node deployment has been discussed and analyzed within the circular shape coverage region under two dissimilar strategies. Such deployment strategies are hexagon based equilateral triangle tessellation deployment and proposed new probabilistic model for coverage improved disc shape deployment.

\section{HEXAGON SHAPE DEPLOYMENT STRATEGY}

According to the properties of the Omnidirectional antenna, the communication range of the sensor node has been presumed as a circle with radius ' $R c^{\prime}$ ', and the coverage region of interest is a reckoning as a

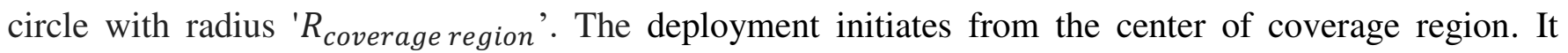
means the first node should be deployed precisely in the center of the circle. Remaining nodes are deployed on inner hexagons within the coverage region. Hexagon based deployment is similar to the equilateral triangle tessellation based deployment. The size of the inner hexagon is evenly increased with the steps of ' $(\sqrt{3}) \times R s^{\prime}$. Relationship between the side (D) of inner hexagon and radius of sensing range $(R s)$ is:

$$
D=n(\sqrt{3}) \times R s
$$

Where, $\mathrm{n}=1,2,3,4, \ldots$.

According to the structure of hexagon, the angle between vertexes will be $60^{\circ}$. Relationship between the area of hexagon shape $\left(A_{H}\right)$ and area of the circle shape $\left(A_{C R}\right)$ is given by:

$$
\begin{aligned}
& A_{H-n}=\left[3(\sqrt{3}) \times D_{n}^{2}\right] / 2 \\
& A_{C R}=\pi \times R_{C R}^{2} \\
& A_{H-n} \leq A_{C R}
\end{aligned}
$$


Where, $R_{C R}=$ Radius of the circle, $A_{H-n}=$ Area of the $\mathrm{n}^{\text {th }}$ hexagon.

Every increment in the size of the inner hexagon requires more number of the sensor node. Relationship between the number of the inner hexagon and the required number of nodes is written as,

$$
N_{H-n}=6 \times n
$$

Where, $\mathrm{n}=1,2,3,4, \ldots, N_{H-n}=$ total number of nodes in $\mathrm{n}^{\text {th }}$ hexagon.

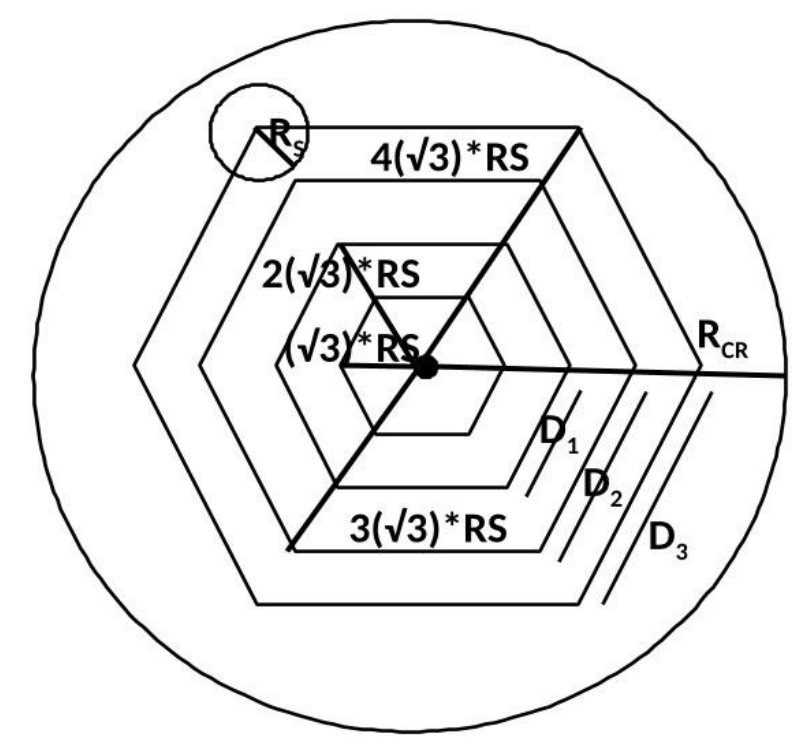

(a)

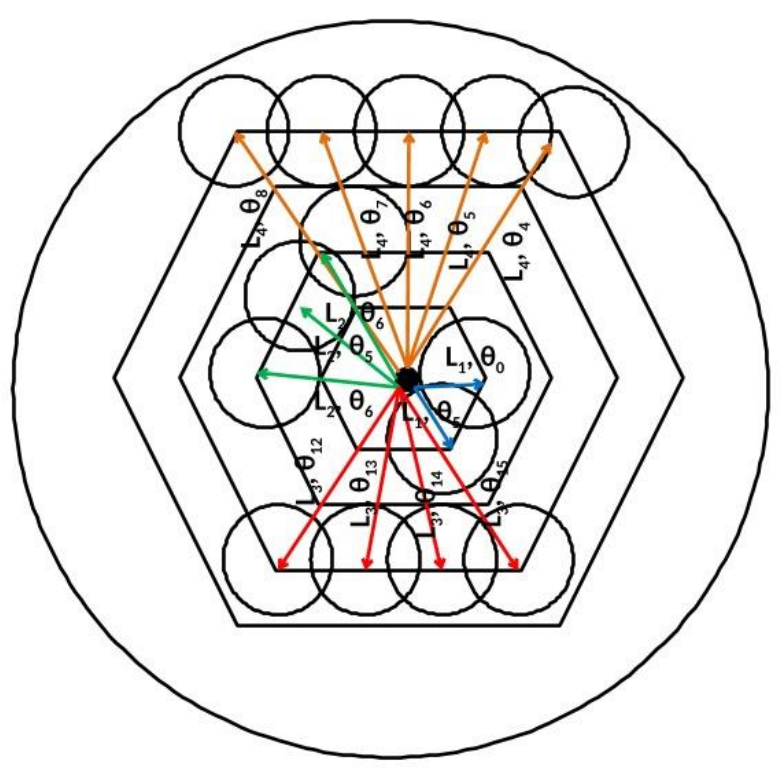

(b)

FIGURE 7 Geometry for sensor node deployment in circular coverage region. (a) Scale for hexagon shape deployment. (b) Angle specification for hexagon shape deployment 
Sensor node deployment on vertexes and side of the hexagon can be preceded by proper length ' 1 ' and angle ' $\theta$ ' from the origin. The geometry of a hexagon shape deployment strategy is shown in Figure 7. For example, if the first hexagon from the origin is considered, each vertex will be occupied by one sensor node. It reveals that the entire area surrounded by inner hexagon is getting covered. It does not require any supplementary sensor node on the side of the hexagon to consummate the coverage. In the second hexagon from the origin, the coverage hole present between vertexes after the deployment of a sensor node at each vertex. It requires one more sensor node on each side to coverage the area surrounded by the second hexagon. Similarly, every increment in the size of the hexagon 'D' requires an increased number of a sensor node on each side (between vertex) as shown in Equation 7. This process is sustained until it reaches full coverage about the entire area of a circular coverage region. Algorithm for hexagon based equilateral triangle tessellation is given below:

\section{Algorithm for Hexagon based deployment}

$$
\begin{aligned}
& \text { for }\left(p=1 ; p \leq D_{n} ; p^{++}\right) \\
& \{ \\
& m=3 \times p ; \\
& \text { for }\left(q=1 ; q \leq p ; q^{++}\right) \\
& \{ \\
& \text {for }\left(n=1 ; n \leq 6 ; n^{++}\right) \\
& \{ \\
& \theta_{q}=q \times\left(\frac{60^{\circ}}{p}\right) \\
& l_{q}=\sqrt{ }\left[(m /(p+1-q))^{2}+m^{2}-\left(2 \times\left(\frac{m}{p+1-q}\right) \times m \times \cos \left(60^{\circ}\right)\right] ;\right. \\
& \text { if }(n=1) \\
& \{ \\
& \theta_{n}=\theta_{q} \\
& {\left[X_{p}, Y_{p q n}\right]=\left[l_{q} \times \cos \left(\theta_{n}\right), l_{q} \times \sin \left(\theta_{n}\right)\right] ;}
\end{aligned}
$$




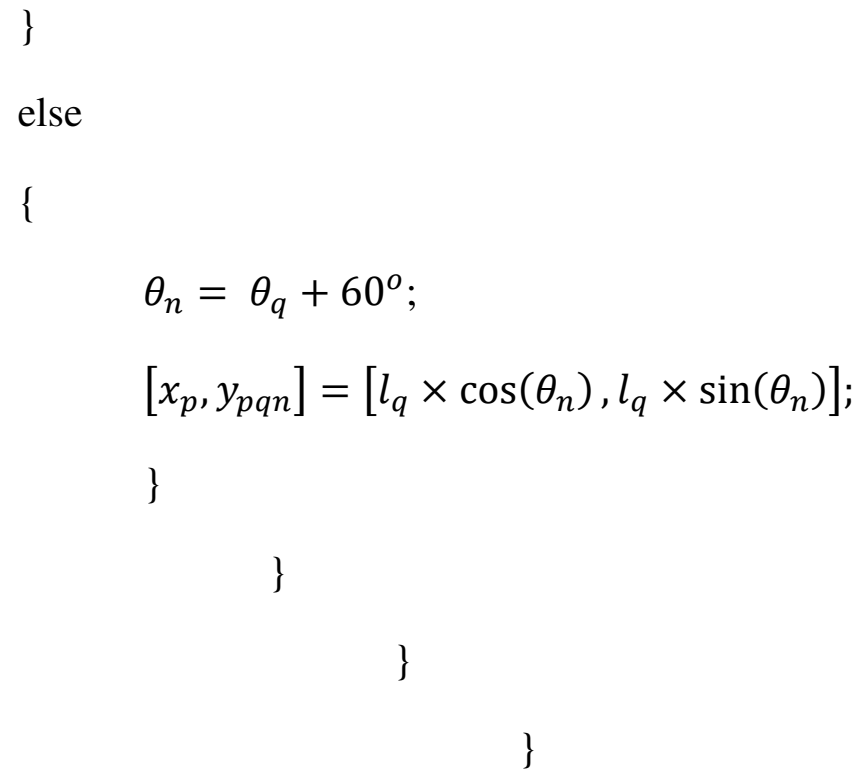

Simulation result in Figure 9 shows that, there is a huge coverage hole between the boundary of coverage region of interest and boundary of ' $\mathrm{n}^{\text {th }}$, hexagon (the last hexagon which is placed within the coverage region). There is no proper algorithm to add supplementary sensor nodes to cover the bare area.

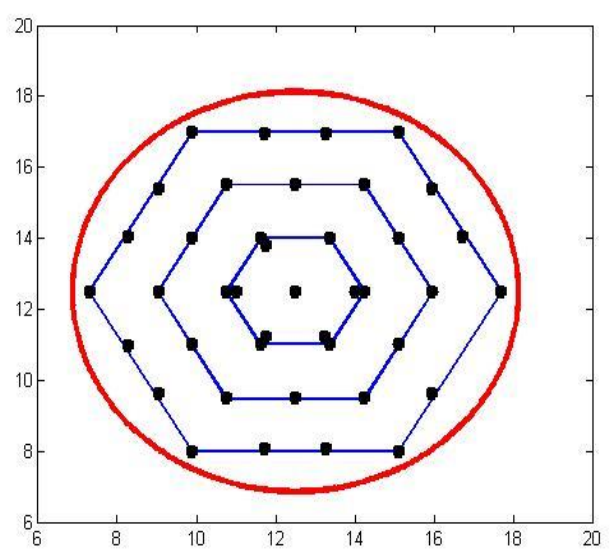

(a)

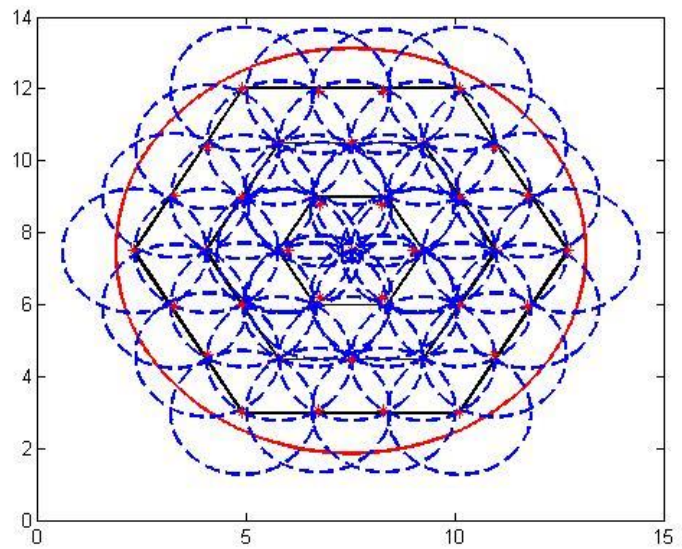

(b)

Figure 8 Disc shape deployment for coverage region with radius 5.642. (a) Hexagon shape deployment without sensing disc. (b) Hexagon shape deployment with sensing disc

As per the hexagon based deployment strategy, each vertex of ' $\mathrm{n}^{\text {th }}$ ' hexagon reaches the boundary at coverage region. It produces coverage hole nearby each side of the hexagon. It requires deploying some supplementary sensor nodes at the bare area to fulfill the coverage. Otherwise, if one more hexagon is 
included, each corner of the ' $\mathrm{n}^{\text {th }}$, hexagon exceeds beyond the circular coverage region, and it is not effective deployment in a wireless sensor network.

Coverage hole is the biggest problem in equilateral triangle tessellation deployment, which requires less number of nodes. To avoid such a coverage hole problem, probabilistic model is proposed for disc shape deployment strategy.

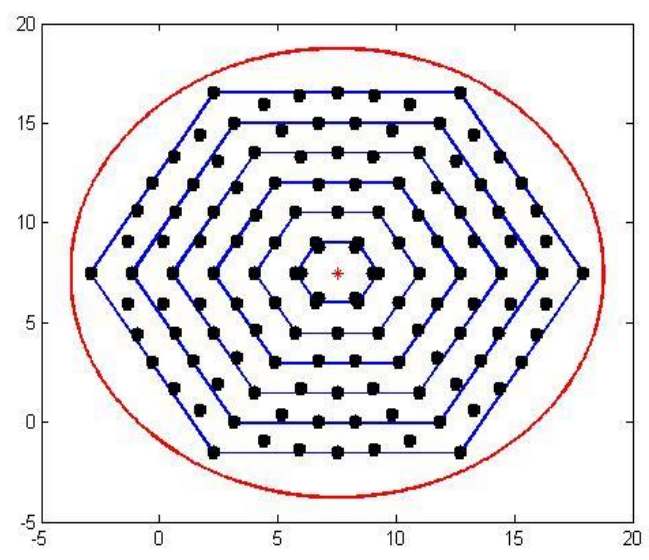

(a)

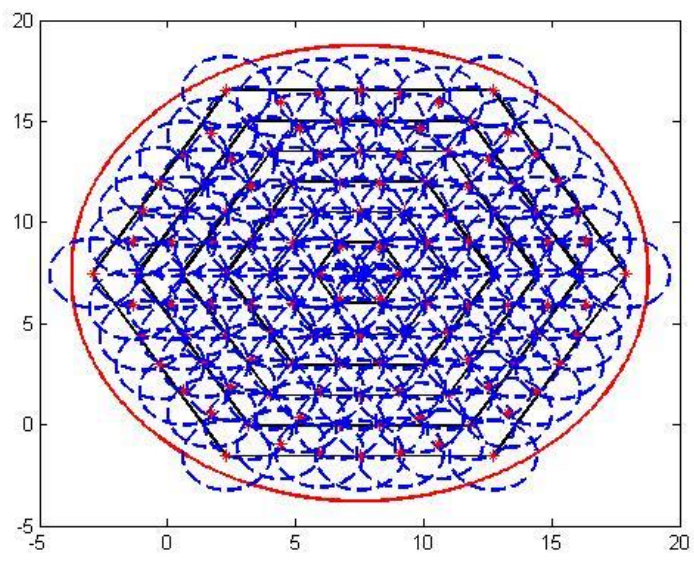

(b)

Figure 9 Disc shape deployment for coverage region with radius 11.284. (a) Hexagon shape deployment without sensing disc. (b) Hexagon shape deployment with sensing disc

\section{PROBABILISTIC MODEL FOR DISC SHAPE DEPLOYMENT STRATEGY}

In the Omnidirectional antenna, the communication range is presumed as a circle. Here, the coverage region of interest is conjectured as a circle with radius ' $R_{\text {(Coverage Region) }}$ '. The deployment of the sensor node begins from the center of the circular coverage region. It means the first node could be placed precisely center of the circle. Remaining node deployment scaled with the help of inner disc with radius ' $n \times(\sqrt{3}) \times R s$ ', where $\mathrm{n}=1,2,3,4, \ldots$

$$
R_{(\text {Dinner }-n)}=n \times(\sqrt{3}) \times R s
$$

Where, $R_{(\text {Dinner }-n)}-$ radius of the $\mathrm{n}^{\text {th }}$ inner disc 
The probability to assume the coverage region of interest is:

$\operatorname{Prob.}\left(C R_{(\text {Coverage-Region })}\right)=\left\{\begin{array}{c}C R_{(\text {Coverage-region })}, R_{C R} \leq R_{(\text {Dinner-last })}+\frac{(\sqrt{3}) \times R s}{2} \\ 0, \text { otherwise }\end{array}\right.$

Where, $R_{\text {(Coverage-Region) }}$ is a circular coverage region, $R_{C R}$ is the radius of circular coverage region, $R_{(\text {Dinner-last })}$ is the radius of last inner disc.

The probability to place inner disc within the coverage region is:

$$
\operatorname{Prob} .\left(D_{\text {inner }}\right)=\left\{\begin{array}{c}
D_{\text {inner-n }}, R_{(\text {Dinner }-n)} \leq R_{C R} \\
\text { end, otherwise }
\end{array}\right.
$$

Where, $D_{\text {inner }}$ is the inner disc, $R_{(\text {Dinner }-n)}$ is Radius of the $\mathrm{n}^{\text {th }}$ inner disc

Probability of angle deviation $(\theta)$ to place sensor nodes on hexagon is:

$$
\operatorname{Prob} .(\theta)=\left\{\begin{aligned}
\theta_{n}, & \theta \leq 360^{\circ} \\
\text { end, } & \text { otherwise }
\end{aligned}\right.
$$

Each inner disc is equally divided by:

$$
D_{(\text {Dinner }-n)}=\frac{2 \times \pi}{8 \times n}
$$

Where, $\theta_{(\text {Dinner }-n)}$ is the angle of $n^{\text {th }}$ inner disc $\& n=1,2,3,4, \ldots$

\subsection{Geometry for Disc Shape Deployment}

The presumption of coverage region of interest depends on the radius of the disc. At the same time, the probability of placing an inner disc depends on the Radius of Coverage Region of interest $\left(R_{C R}\right)$. As per the analysis, the radius of coverage region of interest $\left(R_{C R}\right)$ and radius of inner disc $R_{(\text {Dinner })}$ are interrelated with each other.

For example, first inner disc is divided equally by $\frac{2 \times \pi}{8}\left(45^{\circ}\right)$ angle (i.e. inner disc is divided into 8 number of equal steps with $45^{\circ}$ angle) and the second inner disc is divided equally by $\frac{2 \times \pi}{16}\left(22.5^{\circ}\right)$ 
(i.e. inner disc is divided into 16 number of equal steps with $22.5^{\circ}$ angle). Similarly, every increment in inner disc is divided equally by the angle of $\frac{2 \times \pi}{8 \times n}$ (where, $\mathrm{n}=1,2,3,4, \ldots$ )

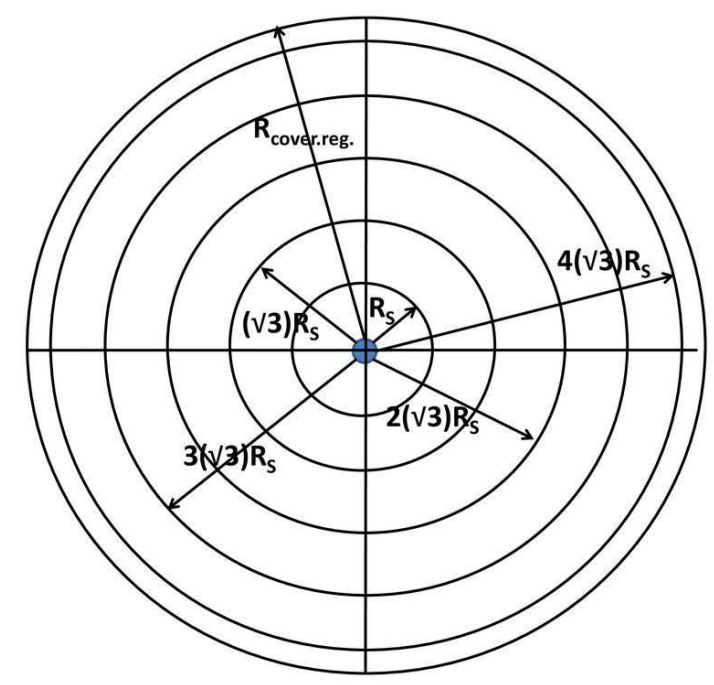

(a)

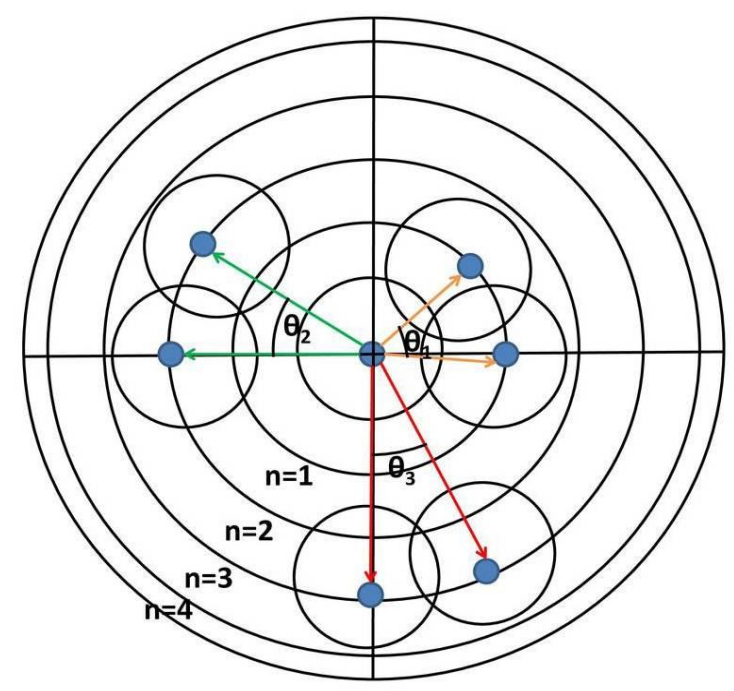

(b)

FIGURE 10 Geometry for sensor node deployment in circular coverage region. (a) Scale for disc shape deployment. (b) Angle specification for disc shape deployment

As per the attribute of the circle, the longest chord traveling through the center of the circle is called diagonal, and half of the diagonal is called the radius of the circle. It is assumed that the first diagonal drawn through the center of the origin is called horizontal diagonal of the circle. The perpendicular line 
which is drawn concerning the horizontal line is called vertical diagonal. According to those presumptions, each inner disc is divided into multiple equal steps. Division starts from $0^{\circ}$ (right end of the horizontal line of each inner disc), and it continues through an anti-clockwise direction. It ends up with $360^{\circ}\left(0^{\circ}\right)$.

The difference in angle between inner discs has been varied based on $\theta_{1}$ as $45^{\circ}, \theta_{2}$ as $22.5^{\circ}$ and $\theta_{3}$ as $11.25^{\circ}$, and it continues till reaching the final disc. The division should be finished with $360^{\circ}$ angle, which means each sensor node should be deployed with $45^{\circ}$ angle deviation on the first inner disc. Deployment starts from $0^{\circ}\left(360^{\circ}\right)$, and it reaches the limit less than $360^{\circ}$. On the whole, the summation of angle difference of sensor node deployment on inner disc $\left(\theta_{11}+\theta_{12}+\theta_{13}+\ldots .+\theta_{1 n}\right)$ should be $360^{\circ}$. The first inner disc contains 8 sensor nodes, second inner disc has 16 nodes, and third inner disc contains 24 nodes. This process continues till reaching the final inner disc, which is placed within the coverage region. The relationship between inner disc and number of the node which is deployed on the inner disc is written as,

$$
N_{(\text {Dinner-n) }}=8 \times n
$$

Where, $N_{(\text {Dinner }-n)}$ is the number of nodes deployed on the $\mathrm{n}^{\text {th }}$ inner disc.

\section{Algorithm for Disc shape deployment}

Step 1: Start

Step 2: Assume ' $\alpha=(\sqrt{3}) \times R s, A=\pi \times r^{2}, R_{C R}=R_{(\text {Coverage-Region })}, R_{\text {Dinner }}=R s, \alpha=(\sqrt{3}) \times R s$ '

Step 3: Place the reference node at the center of the circular coverage region, and assume the center node as an origin.

Step 4: Plot the inner disc with radios ' $p \times a$ ' where $\mathrm{p}=1,2,3,4, \ldots \mathrm{n}$

If $n \times a \leq R_{(\text {Coverage-Region })}$

Plot inner disc

else

Stop

Step 5: Plot the longest diagonal horizontally through the center of the circle. 
Step 6: Plot a perpendicular diagonal concerning horizontal diagonal.

Step 7: Assume each point of the horizontal (forward) line. The horizontal line intersects on the inner disc and coverage region. It is assumed as the starting point of the node deployment.

Step 8: Each inner disc (starting from innermost nearby center) has been equally sub-divided into ' $8 \times p$ ' division with an angle ' $\theta_{p}=\frac{2 \times \pi}{8 \times p}$.

Step 9: Inner disc deployment follows the condition

$$
\begin{aligned}
& K_{q}<360^{\circ}, \text { where } \mathrm{q}=1,2,3, \ldots, \mathrm{m} \\
& \quad \text { if }\left(K_{q} \geq 360^{\circ}\right)
\end{aligned}
$$

The deployment is shifted to the next inner disc.

Step 10: Deployment continues until it reaches 'n' inner disc.

Step 11: Stop

\subsection{Coordinate assignment for Disc shape Deployment}

Simulation results given in Figure 11 and 12 reveals that, there is minimum coverage hole present between the boundary of circular coverage region and boundary of the $\mathrm{n}^{\text {th }}$ inner disc (last inner disc which is placed within the coverage region).

Most of the researchers use the GPS unit as a location-aware system in WSN. Due to the usage of external hardware along with a tiny node in wireless sensor network, bulk energy consumed by the external devices, and it should be reduced to improve the lifetime of the battery. Such kind of boundary aware deployment strategy is beneficial to pre-determine the location of each sensor node, which is deployed within the coverage region of interest.

In deterministic deployment, discovering the coordinates of each sensor node is the dominant task to avoid energy consumption by additionally used location devices (GPS). As per the characteristics of the circle, coordinate of point 'a' with radius ' $\mathrm{R}$ ' and angle ' $\theta$ ' is expressed as,

$$
(x, y)_{C R-a}=R \times \cos (\theta), R \times \sin (\theta)
$$

Where, $(\mathrm{x}, \mathrm{y})_{\mathrm{CR}-\mathrm{a}}$ - coordinate of point 'a' on the circle, $\mathrm{R}$ - radius of the circle. The equation to determine the coordinate based on sensing range with radius ' $\mathrm{R}_{(\text {Dinner-n) }}$ ' and angle ' $\theta$ ' is written as, 


$$
(x, y)_{n m}=R_{(\text {Dinner }-n)} \times \cos \left(m \times \theta_{n}\right), R_{(\text {Dinner }-n)} \times \sin \left(m \times \theta_{n}\right)
$$

Where, $\mathrm{n}=1,2,3,4, \ldots \mathrm{P} ; \mathrm{m}=0,1,2,3,4, \ldots, \mathrm{Q}$.

$$
R_{(\text {Dinner }-n)}=n \times(\sqrt{3}) \times R s
$$

All sensor nodes except the first node in the inner disc follow the above mentioned equations. The coordinate point of each sensor node is ascertained based on the following algorithm.

\section{Algorithm for Coordinate point about Disc shape deployment}

Step 1: Start

Step 2: Initialize ' $\alpha=(\sqrt{3}) \times R s, A=\pi \times r^{2}, R_{C R}=R_{(\text {Coverage-Region })}, R_{\text {Dinner }}=R s, \alpha=(\sqrt{3}) \times$ $R s^{\prime} \mathrm{P}=$ number of inner disc, $\mathrm{q}=$ number of division for each inner disc for deployment.

Step 3: Deploy a reference node at the center of the circular coverage region and presumes center node as the origin.

Step 4: Assign a coordinate ID for each node from the deployment

$$
\begin{array}{ll}
\text { Repeat: } & \text { for }\left(p=1 ; p \leq n ; p^{++}\right) \\
& \theta_{p}=\frac{2 \times \pi}{8 \times p} \\
& R_{(\text {Dinner }-p)}=p \times \alpha
\end{array}
$$

if $\left(\theta_{p} \geq 0\right)$

$$
\begin{aligned}
& \text { for }\left(i=0 ; i \leq u ; i^{++}\right) \\
& \text {for }\left(j=1 ; j \leq v ; j^{++}\right) \\
& \text {for }\left(q=0 ; q<m ; q^{++}\right) \\
& k_{q}=q \times \theta_{p} \\
& \left(x_{i}, y_{j}\right)=\left[R_{(\text {Dinner }-p)} \times \cos \left(k_{q}\right), R_{(\text {Dinner }-p)} \times \sin \left(k_{q}\right)\right. \\
& \text { if }\left(k_{q}<360^{\circ}\right)
\end{aligned}
$$

Repeat

Step 5: Stop 
The geometry of the coverage region of interest is vastly considered to determine the coordinate of the sensor node based on the diverse deployment strategies. Here, each inner disc has been subdivided into numerous slices based on the angle ' $\left[\frac{2 \times \pi}{8 \times n}\right]$ ' degree. Coordinate of the first node which is deployed at the center position of circular coverage region is reckoned as $[0,0]$. Coordinate point of remaining sensor node is given in Table 1 .

TABLE 1 Coordinate point for sensor nodes in disc shape deployment strategy

\begin{tabular}{|c|c|c|c|c|c|c|c|c|}
\hline $\begin{array}{l}\mathrm{D}_{\text {inner }} \\
\text { /node } \\
(\mathrm{x}, \mathrm{y})\end{array}$ & node1 & node 2 & node3 & node4 & node 5 & - & - & - \\
\hline $\begin{array}{l}D_{\text {inner-1 }}-1 \\
\left(45^{\circ}\right)\end{array}$ & $\alpha, 0$ & $\frac{\alpha}{\sqrt{2}}, \frac{\alpha}{\sqrt{2}}$ & $0, \alpha$ & $\frac{-\alpha}{\sqrt{2}}, \frac{\alpha}{\sqrt{2}}$ & $-\alpha, 0$ & $\frac{-\alpha}{\sqrt{2}}, \frac{-\alpha}{\sqrt{2}}$ & $0,-\alpha$ & $\frac{\alpha}{\sqrt{2}}, \frac{-\alpha}{\sqrt{2}}$ \\
\hline $\begin{array}{l}D_{\text {inner-2 }}-2 \\
\left(22.5^{\circ}\right)\end{array}$ & $2 \alpha, 0$ & $\frac{[\alpha \sqrt{2+\sqrt{2}}]}{2}, \frac{[\alpha \sqrt{2-\sqrt{2}}]}{2}$ & $\frac{2 \alpha}{\sqrt{2}}, \frac{2 \alpha}{\sqrt{2}}$ & $\frac{[\alpha \sqrt{2+\sqrt{2}}]}{2}, \frac{[\alpha \sqrt{2-\sqrt{2}}]}{2}$ & $0,2 \alpha$ & \multicolumn{3}{|c|}{ Continue such $22.5^{\circ}$ steps into } \\
\hline $\begin{array}{l}D_{\text {inner-3 }} \\
\left(11.25^{\circ}\right)\end{array}$ & $3 \alpha, 0$ & $2.94 \alpha, 0.6 \alpha$ & $\frac{[\alpha \sqrt{2+\sqrt{2}}]}{2}, \frac{[\alpha \sqrt{2-\sqrt{2}}]}{2}$ & $2.49 \alpha, 1.68 \alpha$ & $\frac{\alpha}{\sqrt{2}}, \frac{\alpha}{\sqrt{2}}$ & \multicolumn{3}{|c|}{$\begin{array}{l}\text { Continue such } 11.25^{\circ} \text { steps } \\
\text { into till } 360^{\circ}(24 \text { nodes })\end{array}$} \\
\hline $\mathrm{D}_{\text {inner }}-\mathrm{n}$ & $n \alpha, 0$ & \multicolumn{7}{|c|}{ Each inner disc divides with $[(2 \pi) / 8 \mathrm{n}]^{\circ}$ steps till it reaches $360^{\circ}$} \\
\hline
\end{tabular}




\subsection{Performance analysis for Disc shape deployment}

The total number of inner discs which are placed within the coverage region of interest is based on the radius of inner discs and radius of coverage region as expressed in Equation 7 . In the proposed deployment strategy, the entire sensor nodes are deployed based on the deployment algorithm given in section 4.2.

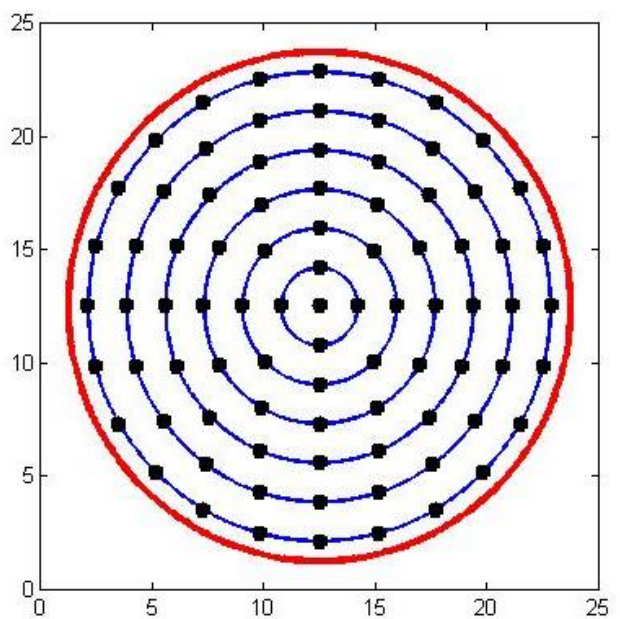

(a)

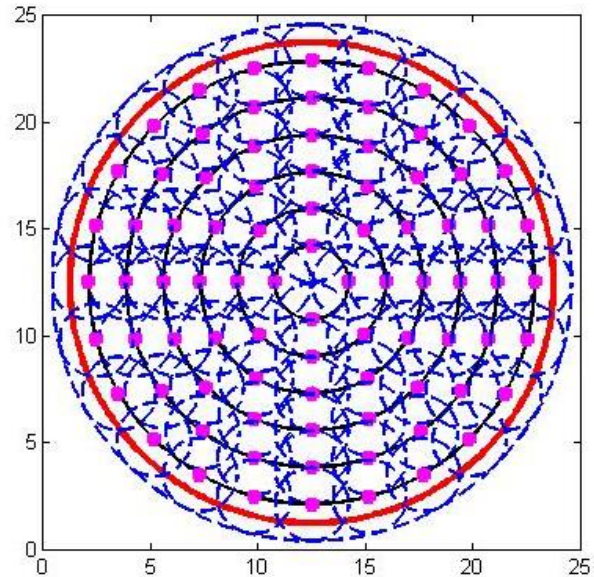

(b)

Figure 11 Disc shape deployment for radius 11.284. (a) Disc shape deployment without sensing disc. (b) Disc shape deployment with sensing disc

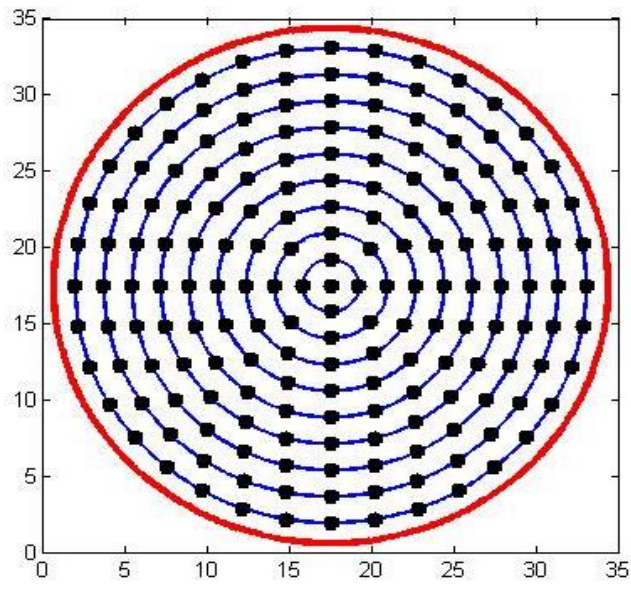

(a)

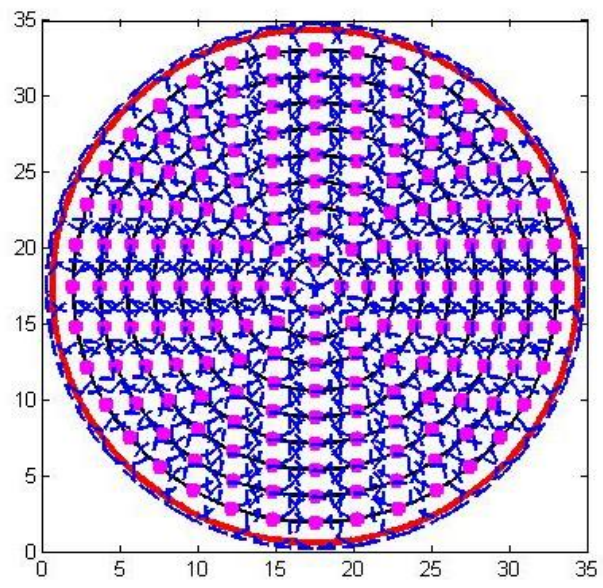

(b)

Figure 12 Disc shape deployment for radius 16.926. (a) Disc shape deployment without sensing disc. (b) Disc shape deployment with sensing disc 
The simulation result manifests that the required number of nodes is vastly varied concerning the coverage region of interest as shown in Figure 11 and 12.

\subsection{Comparative Performance Analysis}

Figure 13 manifests the deployment of sensor nodes in inner disc based on various sensing regions. Comparative analysis has been done between the number of a sensor node required to cover the entire area in square shaped coverage region and proposed circular shaped coverage region.

The area of regular polygon ' $10 \times 10$ ' units square is nearly equivalent to the area of a circle with radius 5.642. Similarly the area of regular polygon ' $20 \times 20$ ' units square is equivalent to the area of a circle with radius 11.284 as listed in Table 2. When compared to the square shaped coverage region, circular shaped coverage region requires lower number of nodes to cover the entire area. For example, 132 nodes are required to cover '10 $\times 10$ ' units of square shaped coverage area, but the circular shape region coverage region requires 84 nodes only. Similarly, 506 nodes are required to cover ' $20 \times 20$ ' units of square shaped coverage region, but circular shaped coverage region requires 364 nodes as plotted in Figure 13.

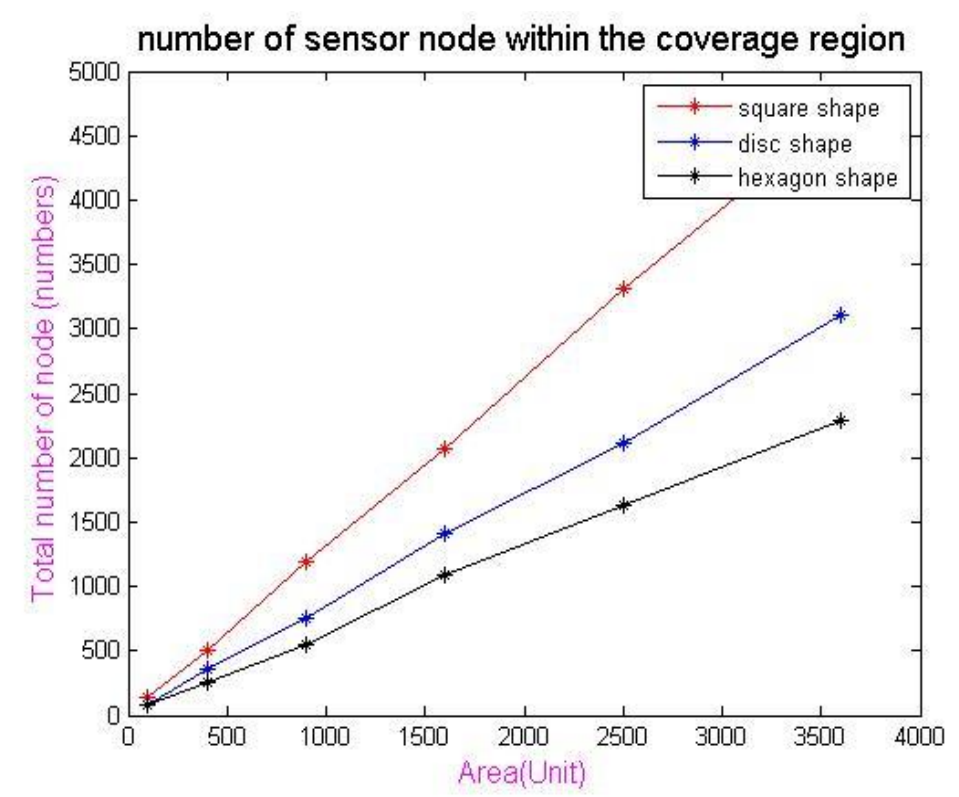

FIGURE 13 Comparison of required nodes for square shape and circular shape coverage regions 
The next simulation also investigated with the help of MATLAB 2014a version, where a comparative analysis has been done about the covered and uncovered area within the circular coverage region of interest under two different deployment strategies known as Hexagon shaped strategy and Disc shaped strategy. The simulation result manifests that the proposed disc shape deployment strategy gives better coverage when compared with the Hexagon shape deployment as listed in Table 2. For example, the uncovered area in disc shape deployment is 1.8 units, but the uncovered area in hexagon shape deployment is 291.1 units. Similarly, the uncovered area in disc shape deployment is 49.4 units, but the uncovered area in hexagon shape deployment is 253.1 units as in Table 2. Proposed disc shape deployment gives preferable coverage in all perspectives.

TABLE 2 Total covered and non-covered area in the circular region

\begin{tabular}{|c|c|c|c|c|c|c|}
\hline \multirow{3}{*}{$\begin{array}{l}\text { Area } \\
\text { (unit) }\end{array}$} & \multicolumn{6}{|c|}{ Circular region } \\
\hline & \multicolumn{3}{|c|}{ Disc shape } & \multicolumn{3}{|c|}{ Hexagon shape } \\
\hline & $\begin{array}{l}\text { Radius } \\
\text { (unit) }\end{array}$ & $\begin{array}{l}\text { Covered } \\
\text { Area }\end{array}$ & $\begin{array}{c}\text { Non-covered } \\
\text { area }\end{array}$ & $\begin{array}{l}\text { Side } \\
\text { (unit) }\end{array}$ & $\begin{array}{c}\text { Area } \\
\text { coverage }\end{array}$ & $\begin{array}{c}\text { Non-coverage } \\
\text { area }\end{array}$ \\
\hline $100(10 \times 10)$ & 5.642 & 84.8 & 15.2 & 5.642 & 75.4 & 24.6 \\
\hline $400(20 \times 20)$ & 11.284 & 398.2 & 1.8 & 11.284 & 291.1 & 108.9 \\
\hline $900(30 \times 30)$ & 16.926 & 850.6 & 49.4 & 16.926 & 646.9 & 253.1 \\
\hline $1600(40 \times 40)$ & 22.568 & 1592.7 & 7.3 & 22.586 & 1339.7 & 260.3 \\
\hline $2500(50 \times 50)$ & 28.210 & 2412.6 & 87.4 & 28.210 & 2023.2 & 476.8 \\
\hline $3600(60 \times 60)$ & 33.851 & 3583.6 & 16.4 & 33.851 & 2846.6 & 753.4 \\
\hline
\end{tabular}




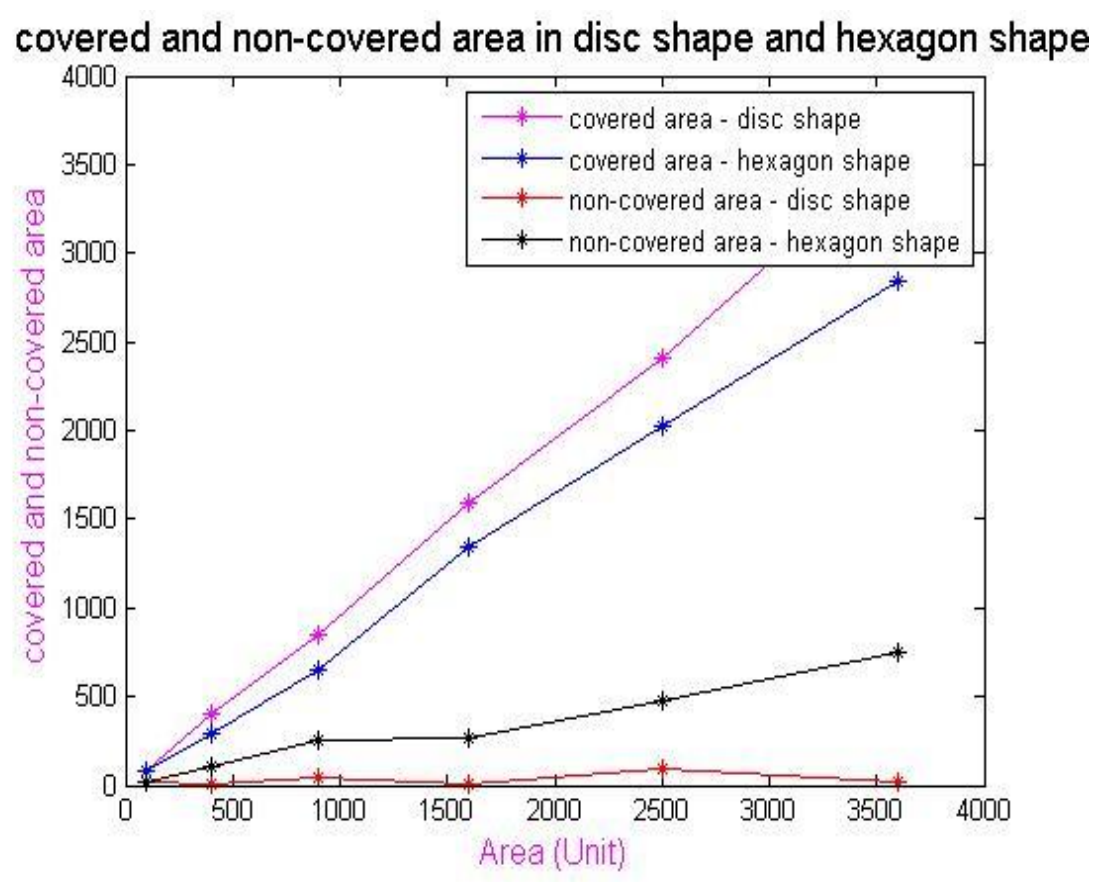

Figure 14 Comparison between Hexagon shape and disc shape deployment strategy based on coverage

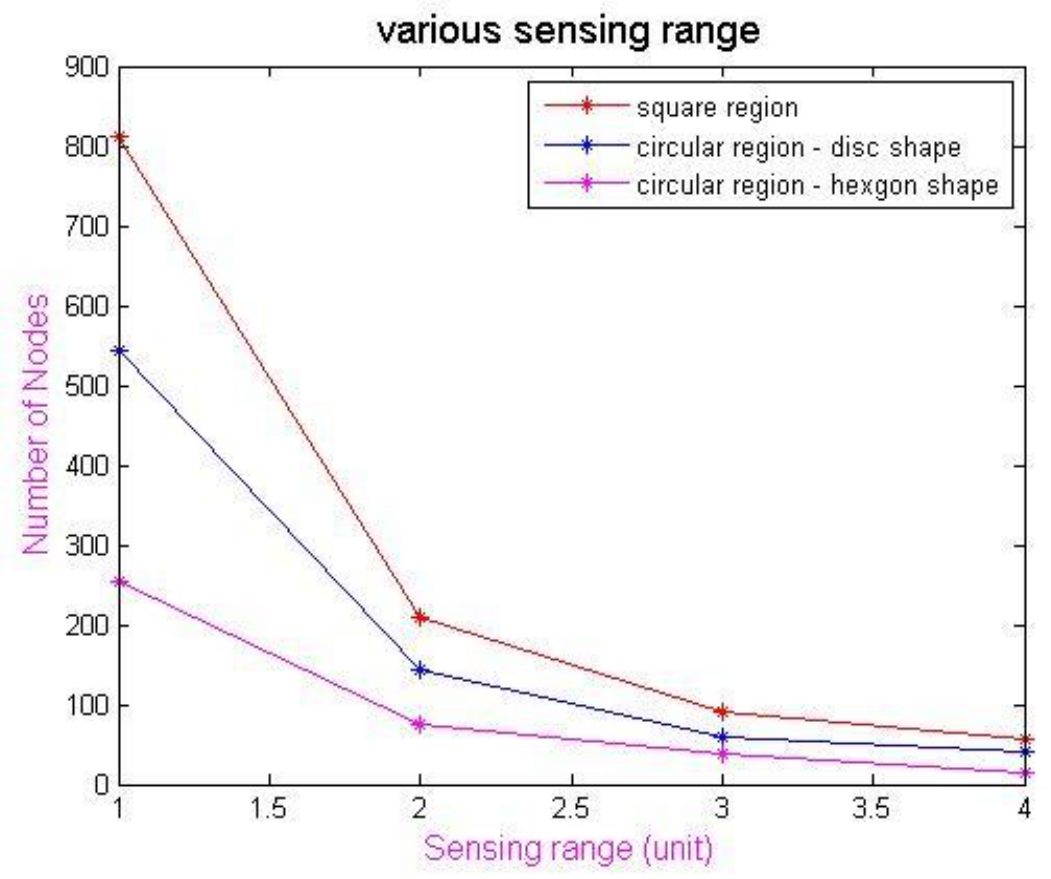

FIGURE 15 Comparison of required nodes in square and hexagon coverage regions based on various sensing range

The communication range can be $\sqrt{3}$ ' time of sensing range. The result manifests that the required number of node is varied based on the sensing range to cover the area of interest. For 
example, when the sensing range is 1 unit, the required number of sensor nodes is 812 to cover the entire square shape coverage region of interest $(50 \times 50$ units), but 544 sensor nodes (disc shape) are required to cover the entire circular coverage region of interest (28.210 units). Similarly, if sensing range is 2 units, the required number of sensor nodes will be 210 to cover the entire square shaped coverage region $(50 \times 50$ units), but 144 sensor nodes (disc shape) only can be used to cover the entire circular coverage region of interest (28.210 units) as shown in Figure 15.

The total number of sensor nodes required to cover the entire coverage region of interest is vastly varied as plotted in Figure 15. The result manifests that, the circular shape coverage region of interest requires minimum number of sensor nodes when compared with the square shape coverage region of interest, and it reduces the coverage hole problem. The inner disc placed within the circular coverage region of interest has been divided into numerous equal parts with a specified angle $\left(\theta_{n}\right)$. Total number of sensor nodes deployed on each inner disc is based on ' $8 n$ ', where ' $n$ ' is the number of the inner disc from the origin. Therefore, the total number of sensor nodes required to cover the entire coverage region of interest is easily determined.

\section{CONCLUSION}

The selection of coverage region of interest is playing a vital role in sensor node deployment, because a vast difference is present in the required number of nodes in two different coverage regions of interest. According to numerical and simulation analysis, when compared with equilateral triangle tessellation (hexagon shape), the disc shape deployment strategy gives much better coverage. It requires few more sensor nodes than hexagon shape. The result manifests that, when compared with equilateral triangle tessellation (hexagon shape) and square shape deployment strategies, the uncovered area of disc shape deployment is lower. The proposed probabilistic model for disc shape deployment strategy in the circular shape coverage region of interest gives better coverage performance. 


\section{Conflict of Interest Statement:}

On behalf of all authors, the corresponding author states that there is no conflict of interest.

\section{REFERENCES}

1. Akyildiz, IF., Su, W., Sankarasubramaniam, Y. \& Cayirci, E. 2002. A survey on sensor network. IEEE Communication Magazine.40 (8): 102-114.

2. Biagioni, ES. \& Sasaki, G 2003. Wireless sensor placement for reliable and efficient data collection. Proceedings of the 36th Annual Hawaii International Conference on System Sciences. Big island, USA.

3. Tian, D., Nicolas \& Georganas, D. 2004. Connectivity Mintenance and coverage preservation in wireless sensor networks. Canadian Conference on Electrical and Computer Engineering.3 (6): 744-761.

4. Ahmed, N., Kanhere, S.S. \& Jha, S. 2005. Probabilistic coverage in wireless sensor networks. IEEE Conference on Local Computer Networks $30^{\text {th }}$ Anniversary (LCN'05). Sidney, Australia.

5. Chang, R.S. \& Wang, S.H. 2008. Self-deployment by density control in sensor networks. IEEE Transactions on Vehicular Technology.57 (3): 1745-1755.

6. Wang, Y.C., Hu, C.C. \& Tseng, Y.C. 2008. Efficient placement and dispatch of sensors in a wireless sensor network. IEEE Transaction on Mobile Computing.7 (2) 262-274.

7. Chatterjee, P, Ghosh, S.C. \& Das, N. 2017. Load balanced coverage with graded node deployment in wireless sensor networks. IEEE Transaction on Multi-Scale Computing Systems. 3 (2): 100-112.

8. Li, X., Frey, H., Santora, N. \& Stojmenovic, I. 2009. Focused-coverage by mobile Sensor Networks. Mobile Ad hoc and Sensor Systems. IEEE 6th International Conference on mobile Adhoc and Sensor System. Macau, China.

9. Wang, Y.C. \& Tseng, Y.C. 2007. Distributed deployment schemes for mobile wireless sensor networks to ensure multilevel coverage. IEEE Transaction on Parallel Distributed System.19 (9): 1280-1294.

10. Bai, X., Kumar, S., Xuan, D., Yun, Z. \& Lai, T.H. 2006. Deploying wireless sensors to achieve both coverage and connectivity. Proceedings of the 7th ACM International Symposium on Mobile Ad Hoc Networking and Computing. Florence, Italy. 
11. Yu, Z., Teng, J., Li, X. \& Xuan, D. 2013. On wireless network coverage in bounded areas. Proceedings IEEE INFOCOM. Turin, Italy.

12. Fadi Al-Turjman, Wireless Sensor Networks: Deployment Strategies for Outdoor Monitoring, CRC Press, 2018, Chapter 6

13. Habib M. Ammari, Mission-Oriented Sensor Networks and Systems: Art and Science, Springer Nature, 2019, Volume 1

14. Zhang, Y., Xue, D., Wu, C., Peng J.I. \& Cheng, L. 2009. Research of nodes deployment for wireless sensor network in determinstic area. Computer Science and Information Technology, ICCSIT 2009, 2nd IEEE International Conference. Beijing, China.

15. Ku, W.S., Sakai, K. \& Sun, M.T. 2011. The optimal k-covering tag deployment for RFID based localization. Journal of Network and Computer Applications.34 (3): 914-924.

16. Yun, Z., Bai, X., Xuan, D., Jia, W. \& Zhao, W. 2010. Pattern mutation in wireless sensor deployment. Proceedings IEEE INFOCOM. San Diago, USA.

17. $\mathrm{Xu} \mathrm{Lu,} \mathrm{Rongjun} \mathrm{Chen,} \mathrm{Jun} \mathrm{Liu,} \mathrm{Lianglun} \mathrm{Cheng,} \mathrm{Square} \mathrm{partition-based} \mathrm{node}$ scheduling algorithm for wireless passive sensor networks, International Journal of Communication Systems, 2018, 31 (8), e3531

18. Zhang, H \& Hou, J.C. 2005. Maintaining sensing coverage and connectivity in large sensor networks. Adhoc \& sensor wireless network.1 (2): 89-124.

19. Wang, Y.C., Hu, C.C. \& Tseng, Y.C. 2005. Efficient deployment algorithms for ensuring coverage and connectivity of wireless sensor networks. First International Conference on Wireless Internet (WICON'05). Budapest, Hangary.

20. Xiaohua Deng, Zhiyong Yu, Rongxin Tang, Xin Qian, Kai Yuan and Shiyun Liu, An Optimized Node Deployment Solution Based on a Virtual Spring Force Algorithm for Wireless Sensor Network Applications, Signal and Information Processing in Wireless Sensor Networks, MDPI Sensors, 2019, 19 (8), 1817 
Figures

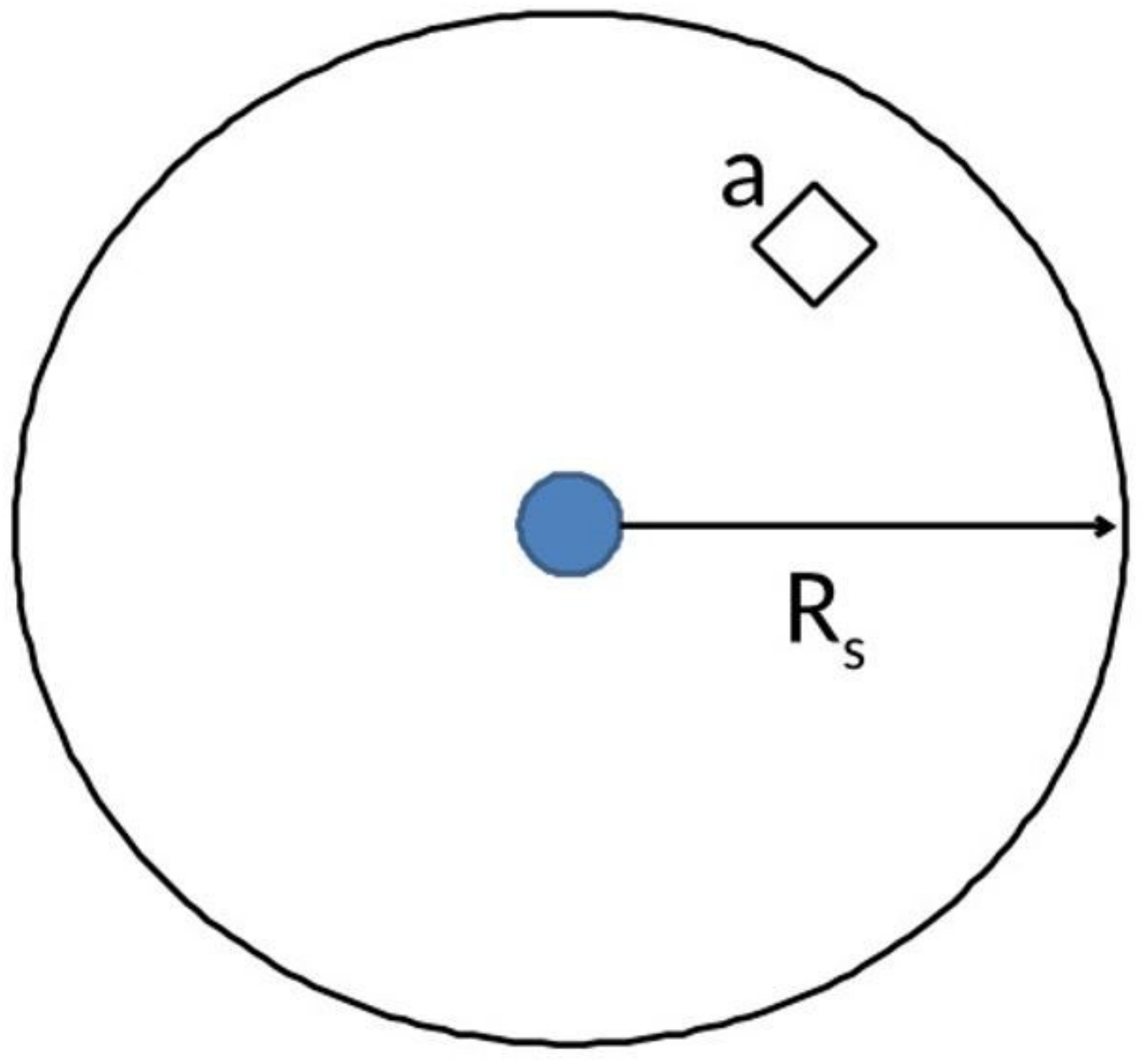

Figure 1

Geometry for sensing model 


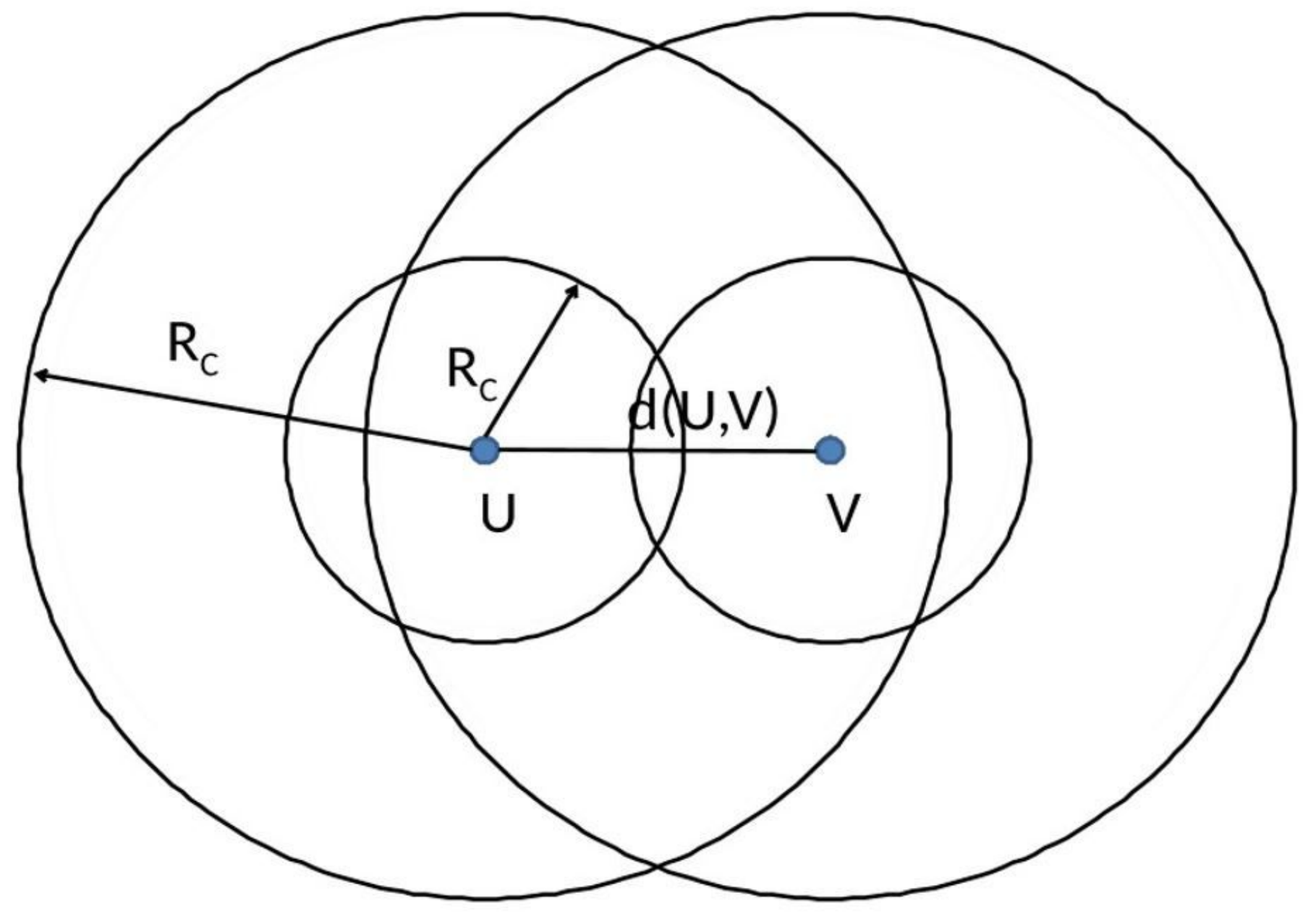

Figure 2

Geometry for a communication model 


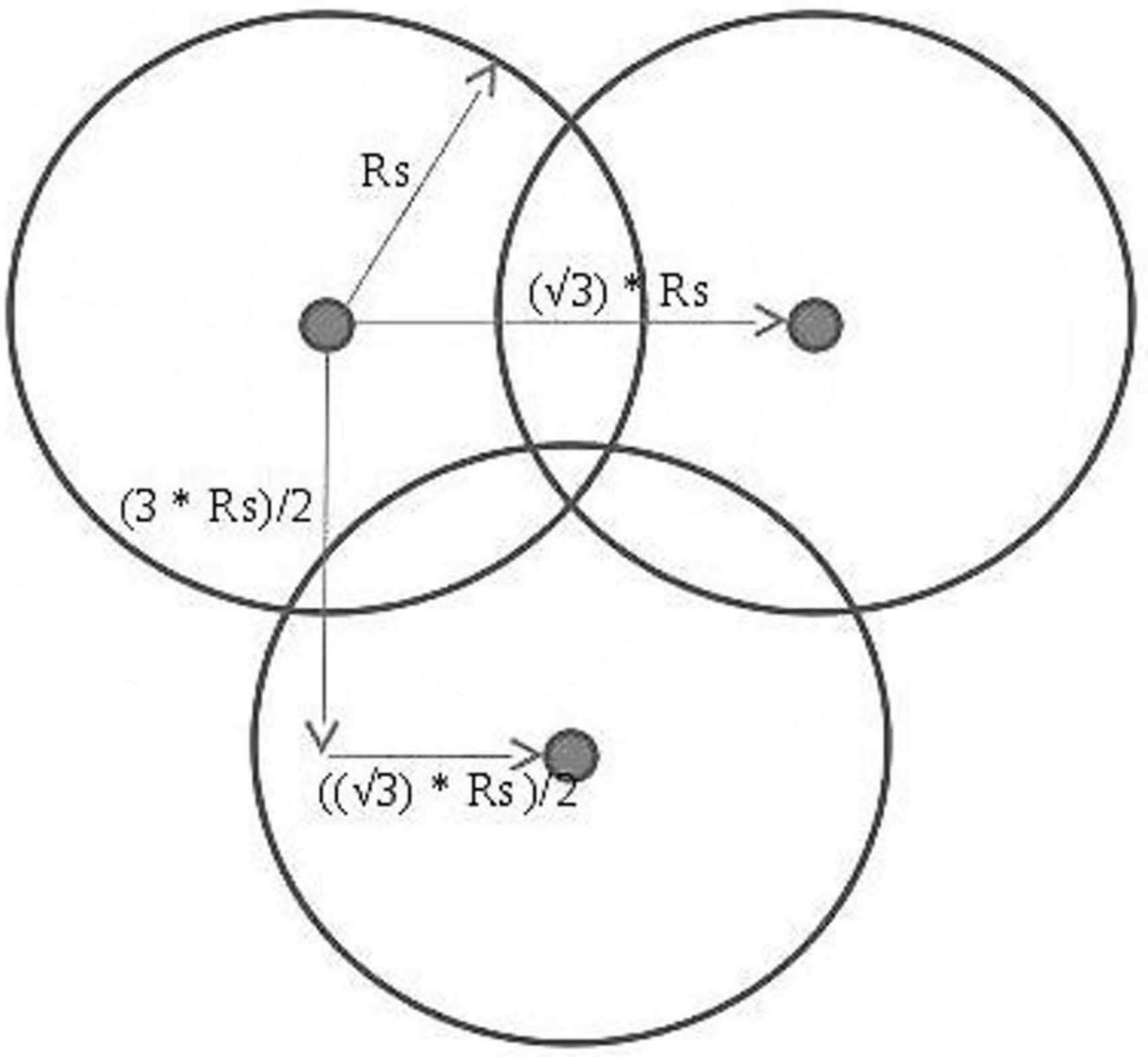

Figure 3

Geometry for strip-based deployment under the condition of Rc $=(\sqrt{ } 3) \times R s$ 


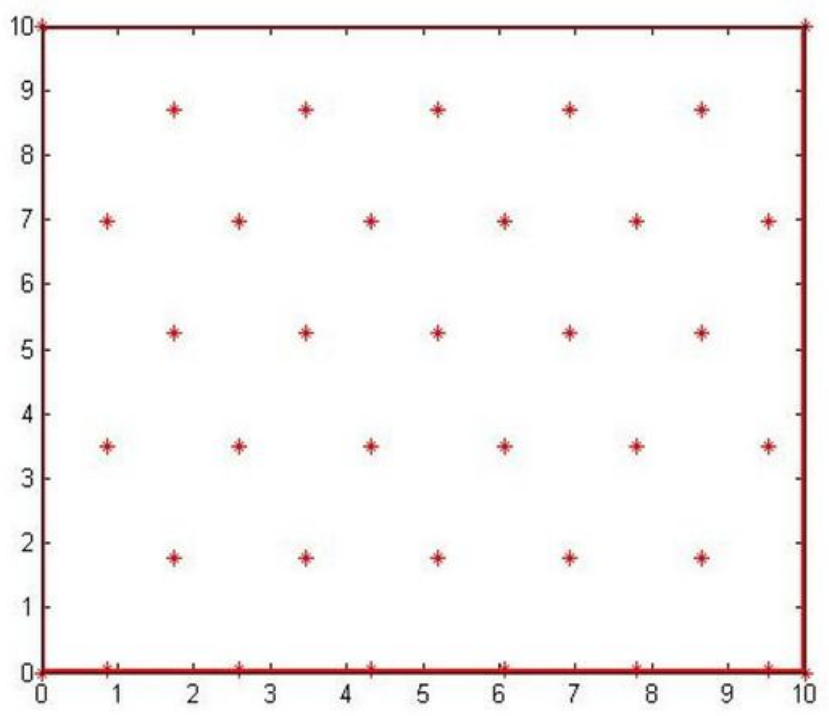

(a)

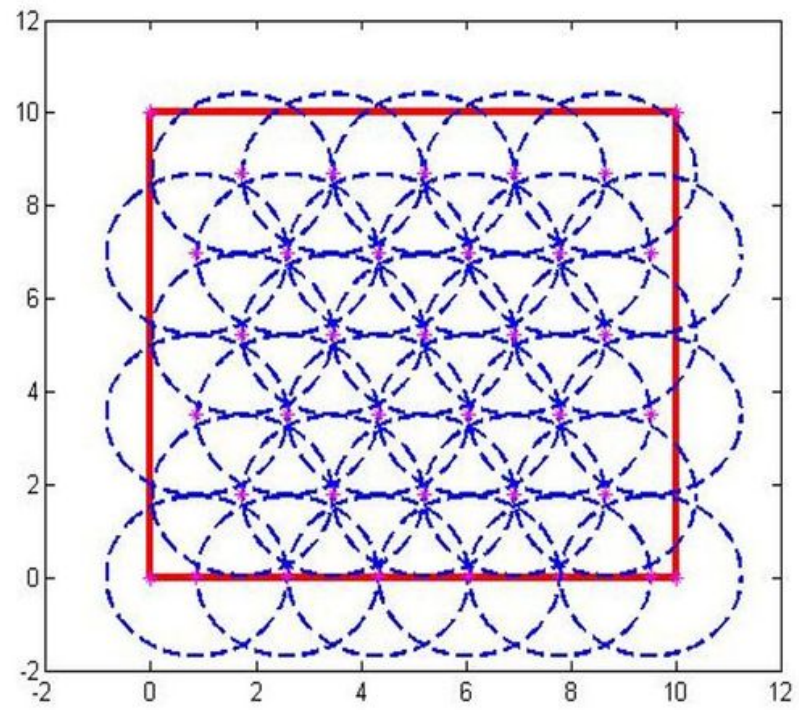

(b)

\section{Figure 4}

Square shape region's strip-based deployment for the geometry of $10 \times 10$ units. (a) Strip based deployment without sensing disc (b) Strip based deployment with sensing disc.

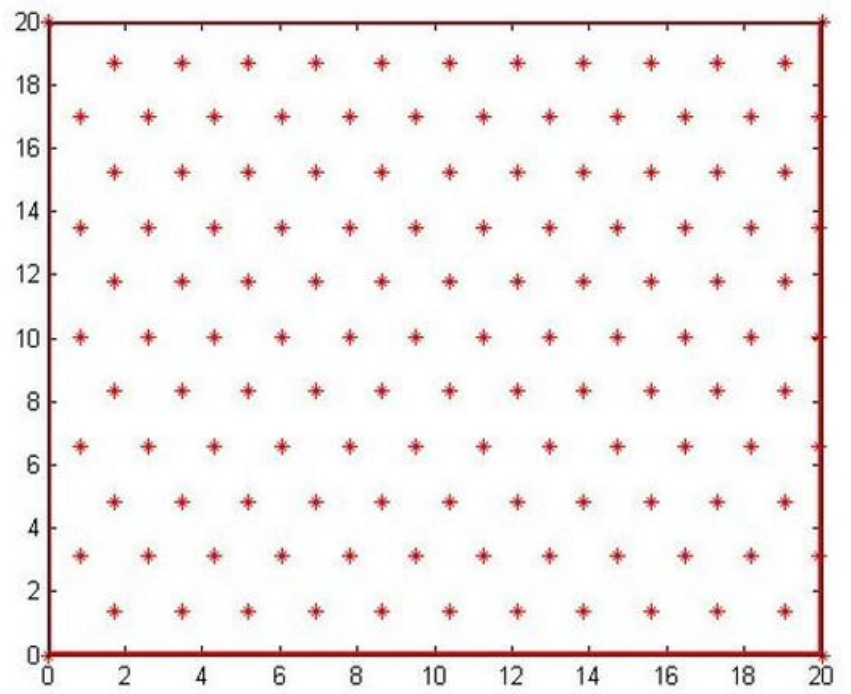

(a)

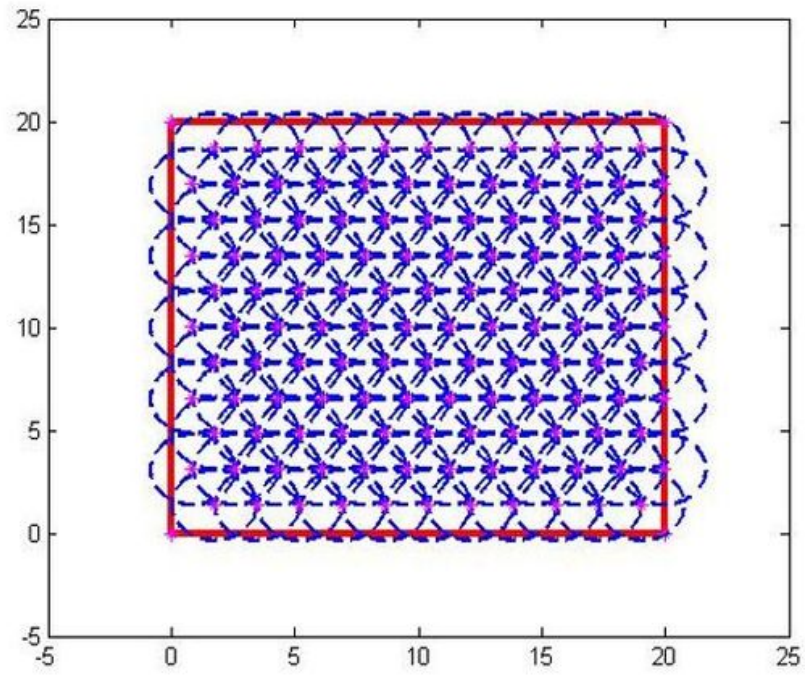

(b)

\section{Figure 5}

Square shape region's strip-based deployment for the geometry of $20 \times 20$ units. (a) Strip based deployment without sensing disc (b) Strip based deployment with sensing disc. 


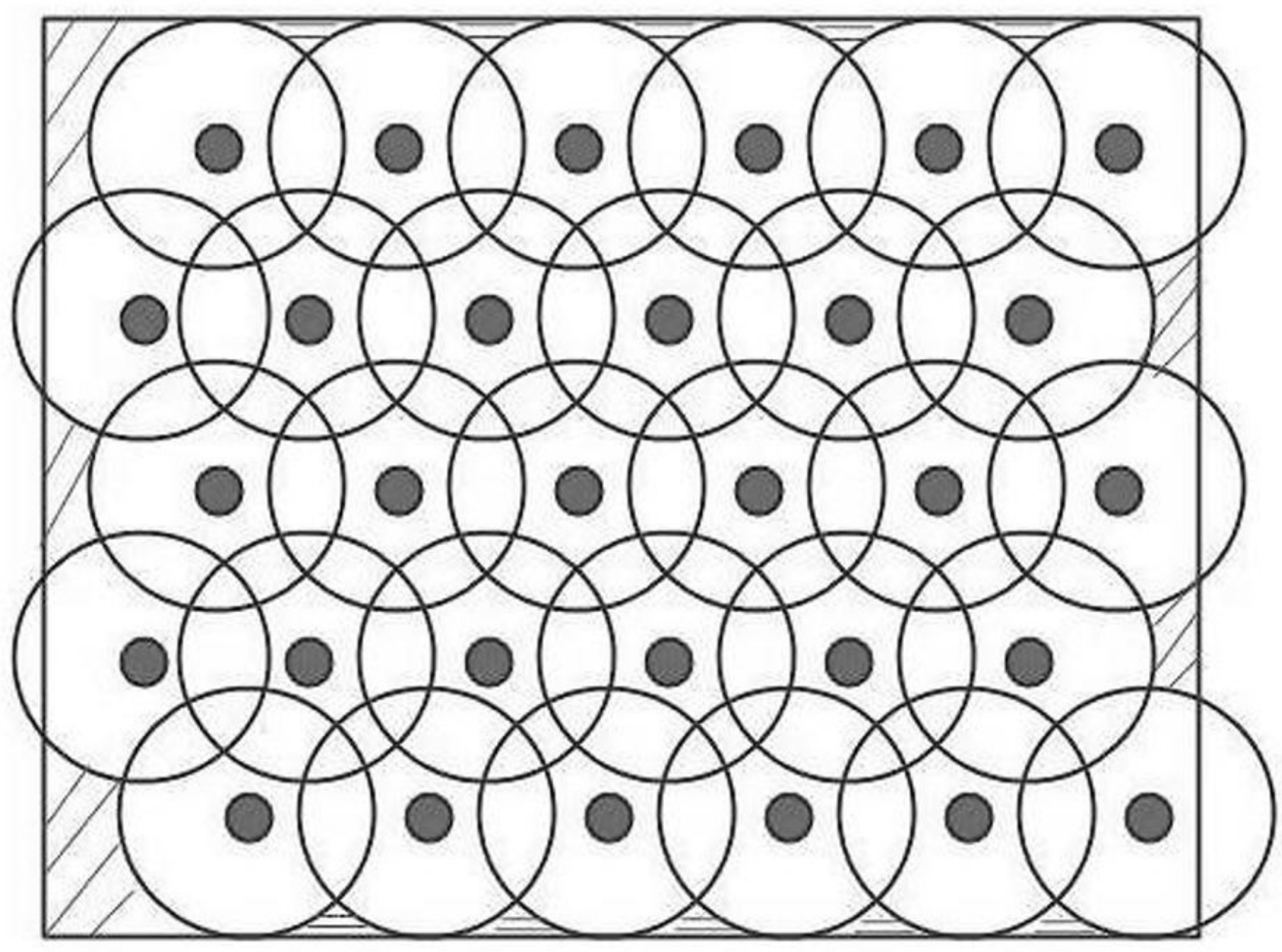

Figure 6

Coverage hole formation in the strip-based deployment. 


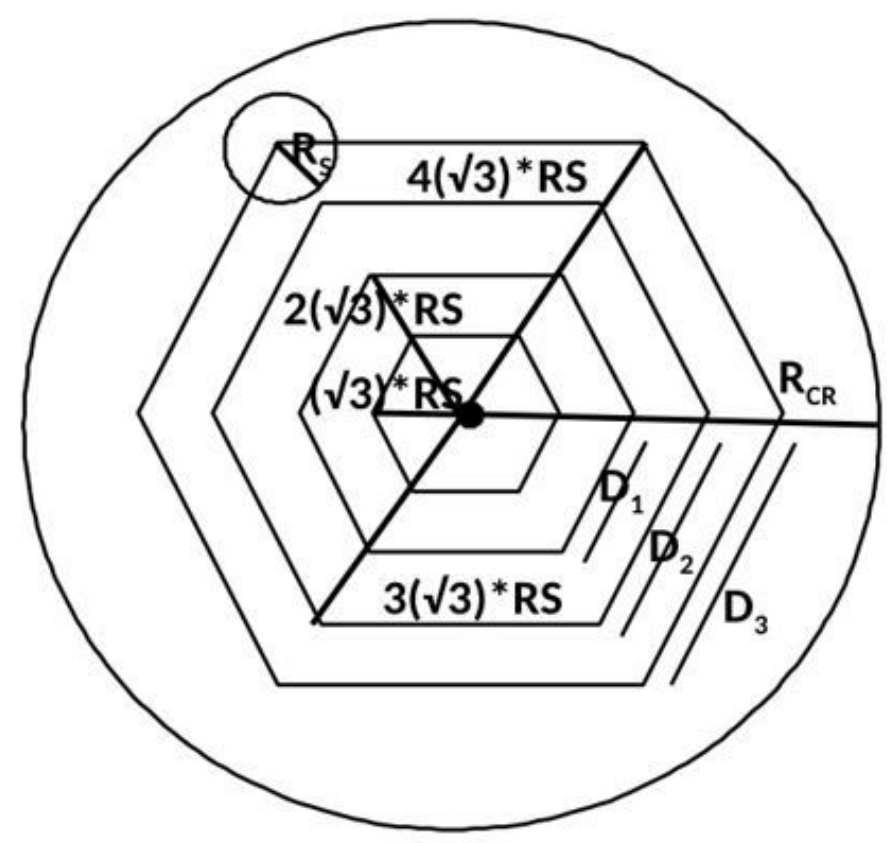

(a)

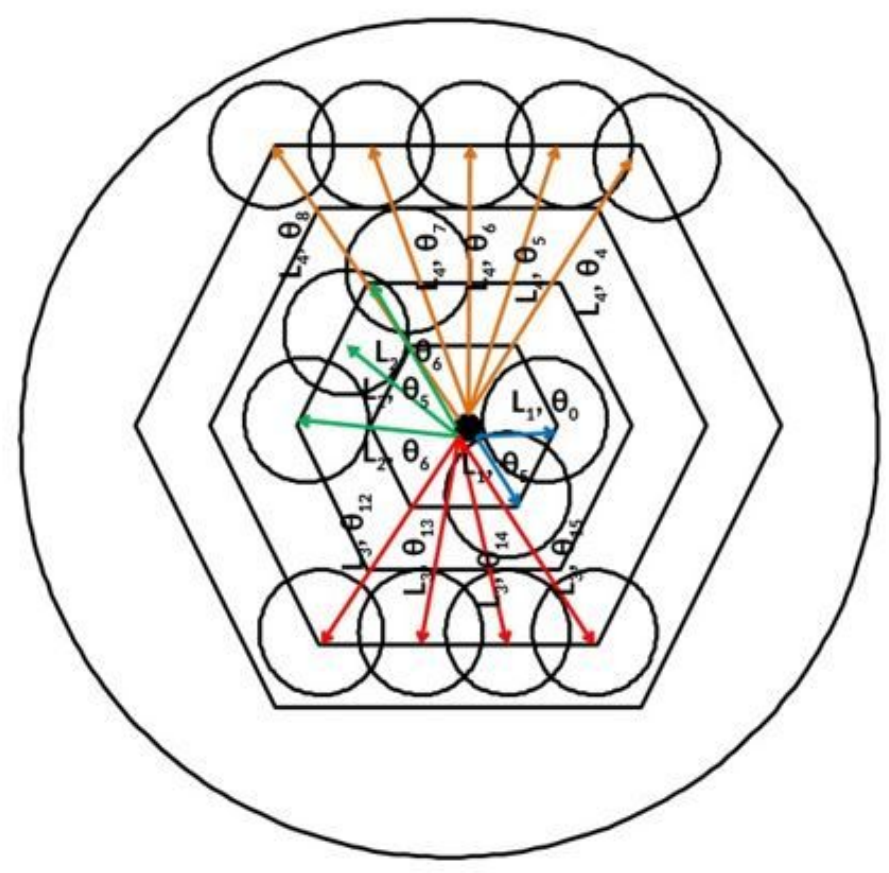

(b)

Figure 7

Geometry for sensor node deployment in circular coverage region. (a) Scale for hexagon shape deployment. (b) Angle specification for hexagon shape deployment 


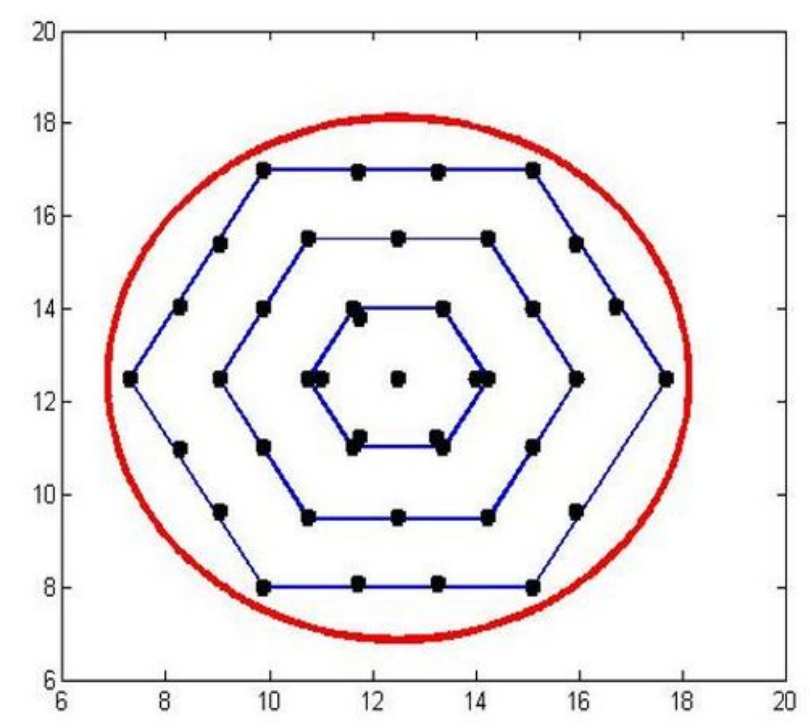

(a)

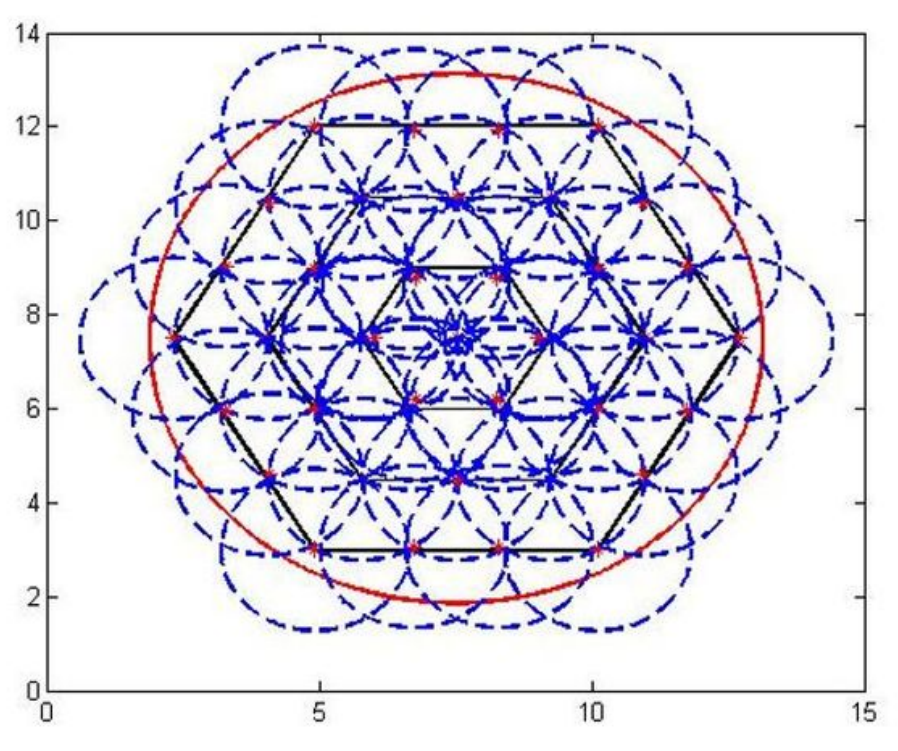

(b)

\section{Figure 8}

Disc shape deployment for coverage region with radius 5.642. (a) Hexagon shape deployment without sensing disc. (b) Hexagon shape deployment with sensing disc

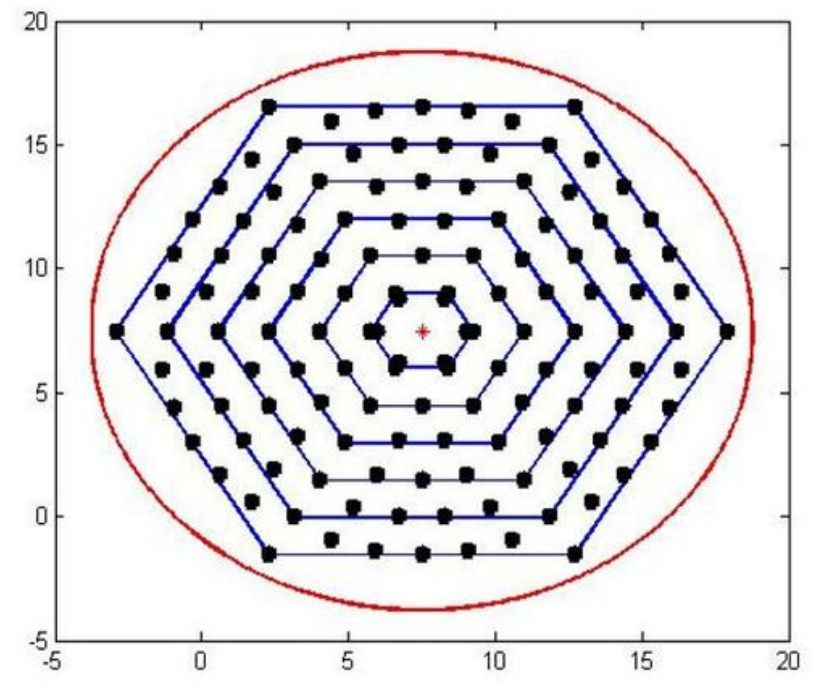

(a)

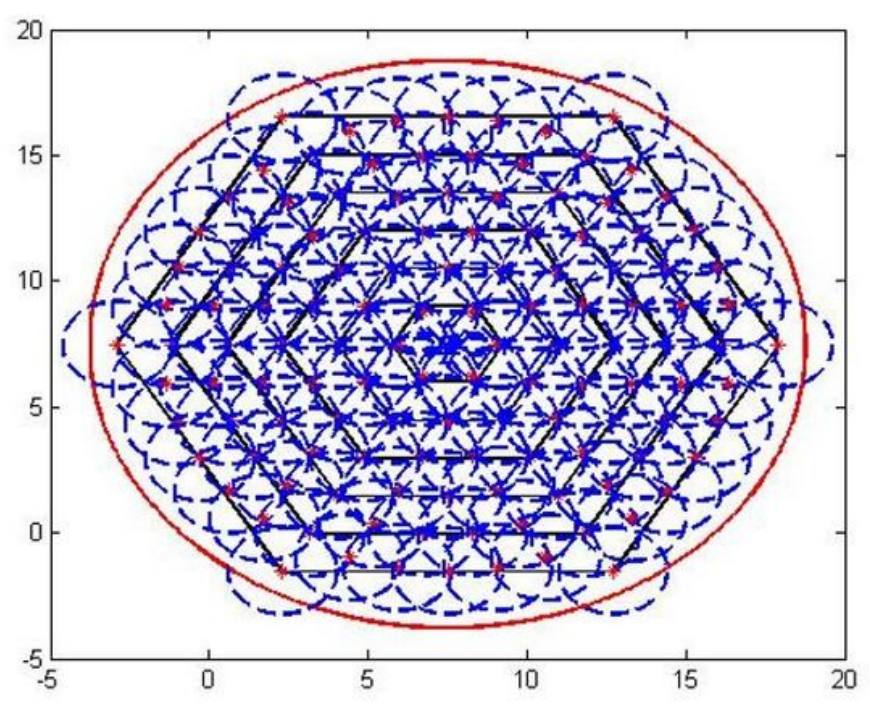

(b)

Figure 9

Disc shape deployment for coverage region with radius 11.284. (a) Hexagon shape deployment without sensing disc. (b) Hexagon shape deployment with sensing disc 


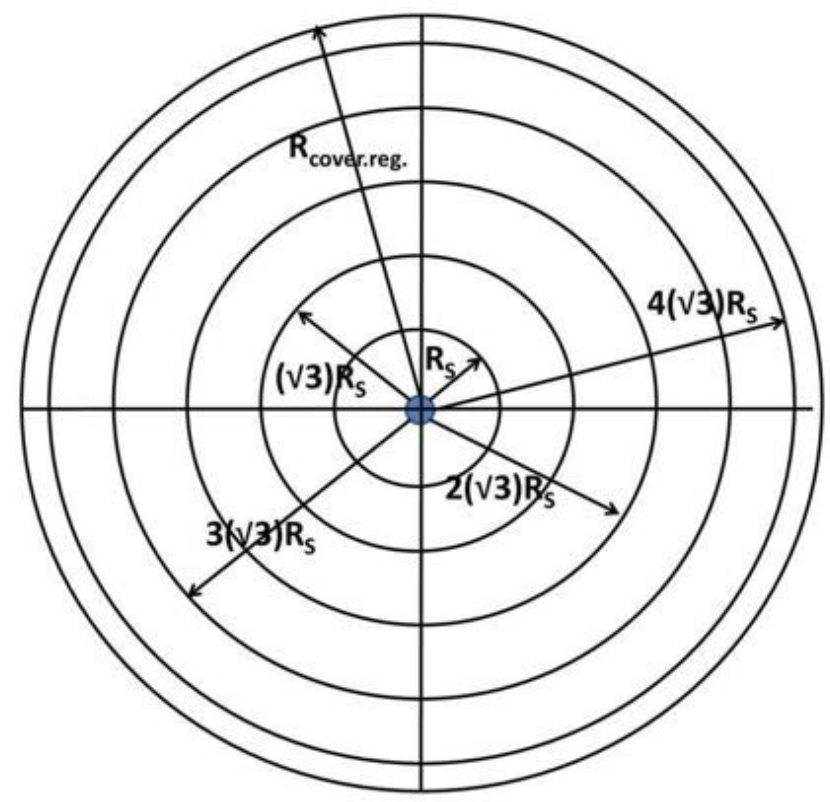

(a)

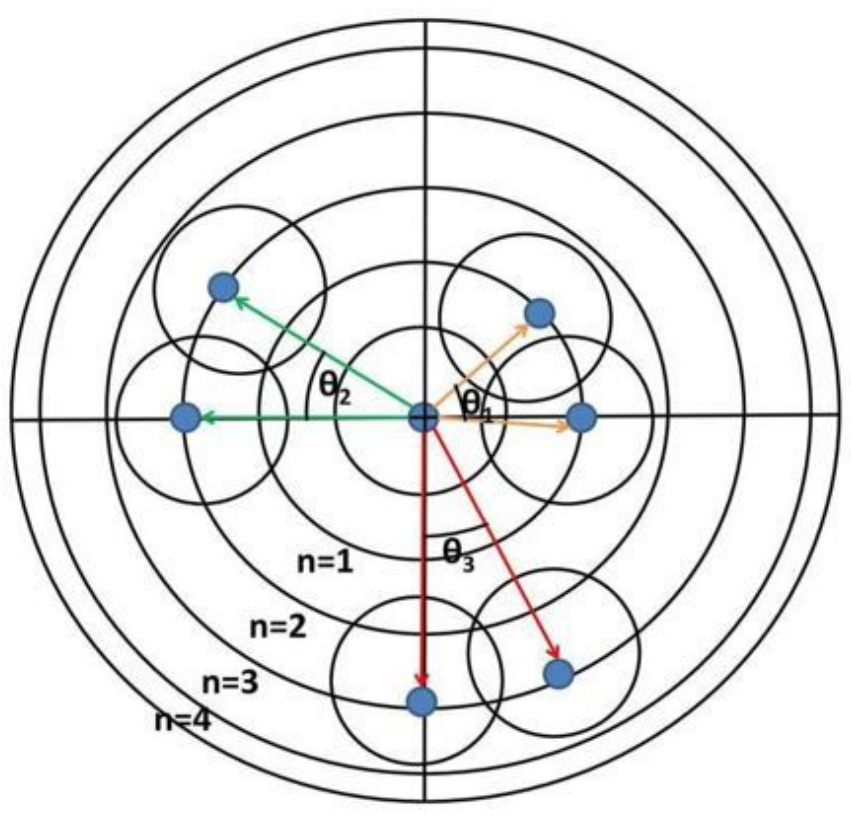

(b)

\section{Figure 10}

Geometry for sensor node deployment in circular coverage region. (a) Scale for disc shape deployment. (b) Angle specification for disc shape deployment 


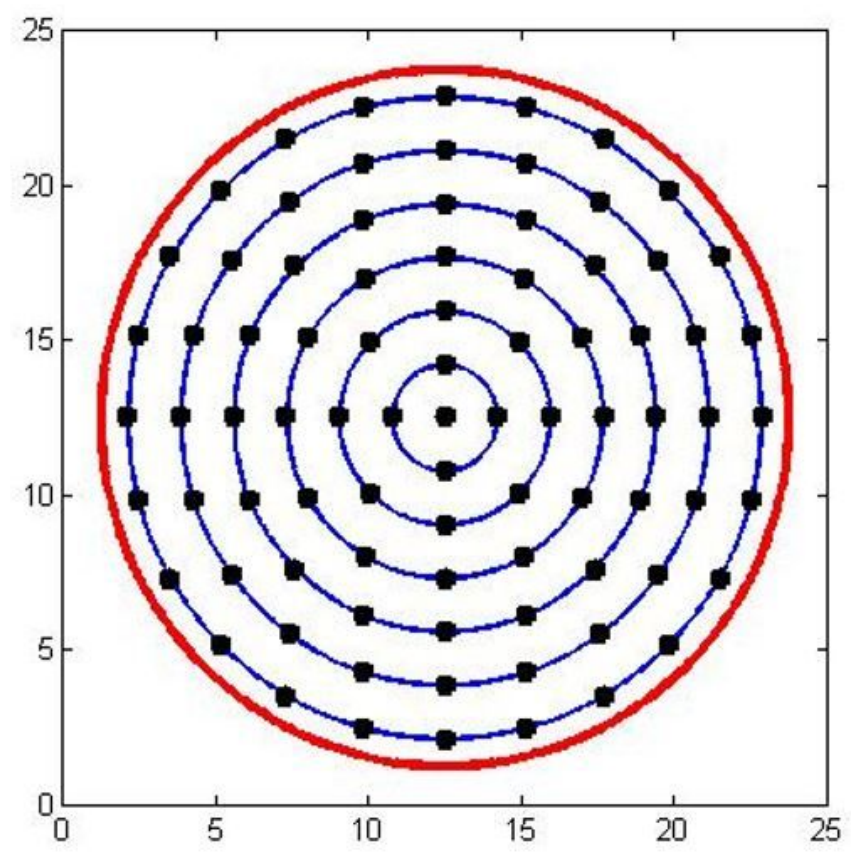

(a)

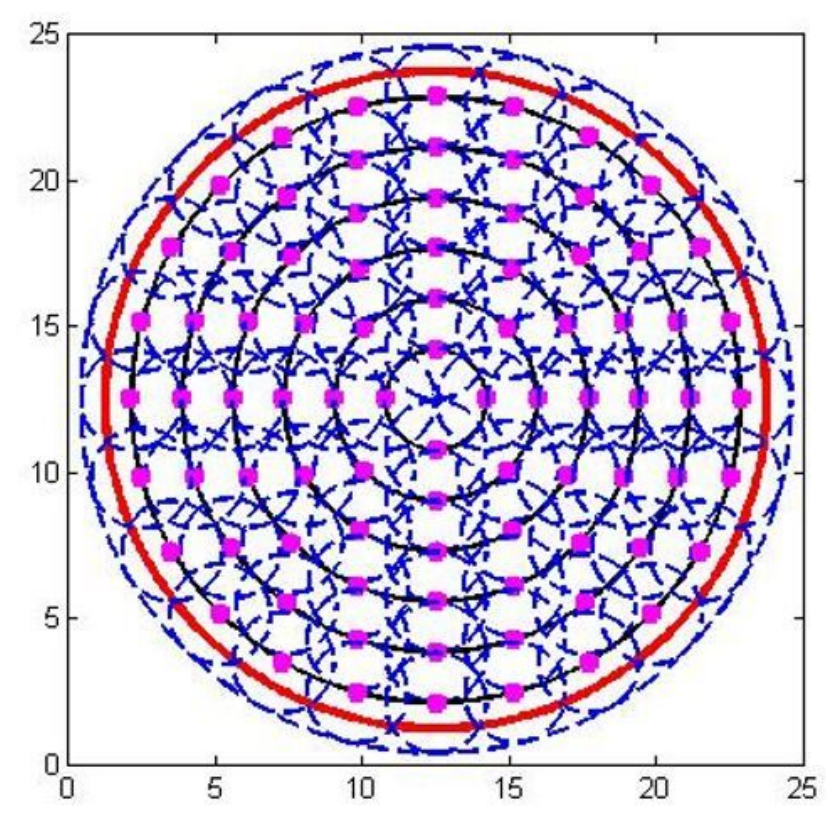

(b)

\section{Figure 11}

Disc shape deployment for radius 11.284. (a) Disc shape deployment without sensing disc. (b) Disc shape deployment with sensing disc 


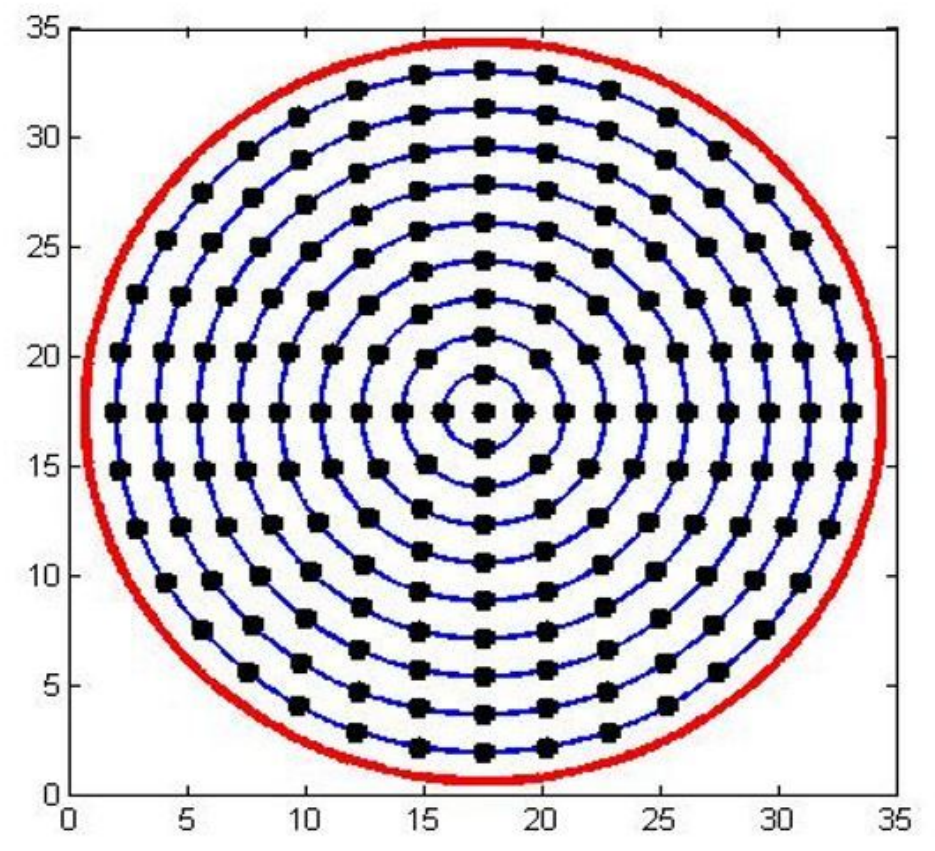

(a)

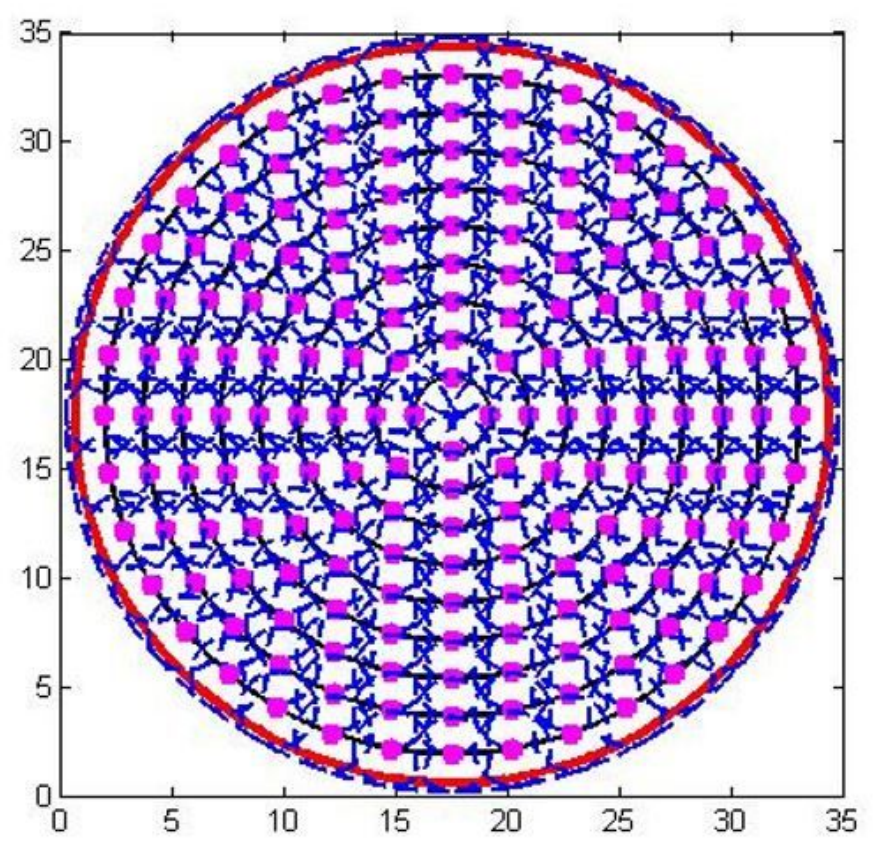

(b)

Figure 12

Disc shape deployment for radius 16.926. (a) Disc shape deployment without sensing disc. (b) Disc shape deployment with sensing disc 


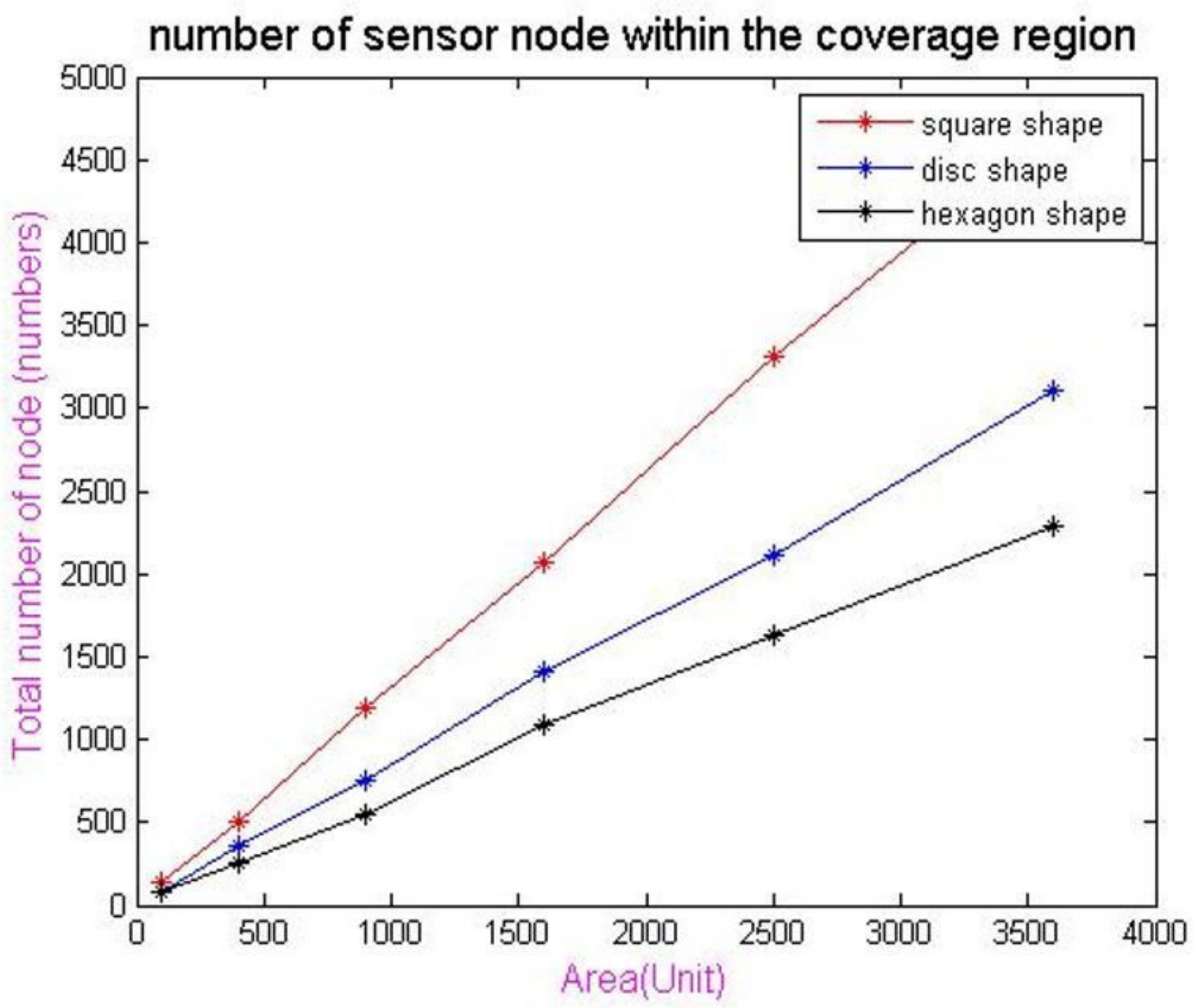

Figure 13

Comparison of required nodes for square shape and circular shape coverage regions 


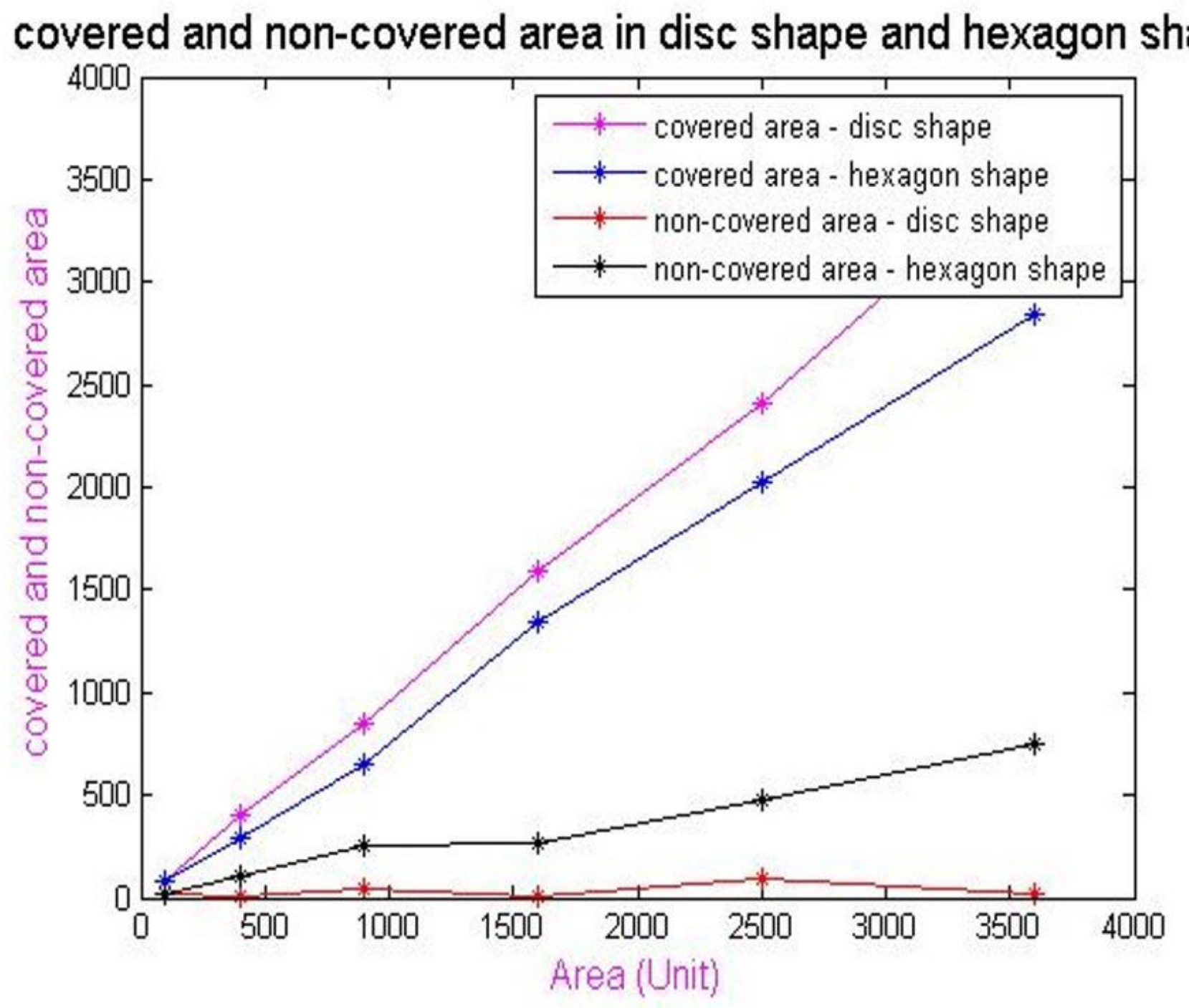

Figure 14

Comparison between Hexagon shape and disc shape deployment strategy based on coverage 


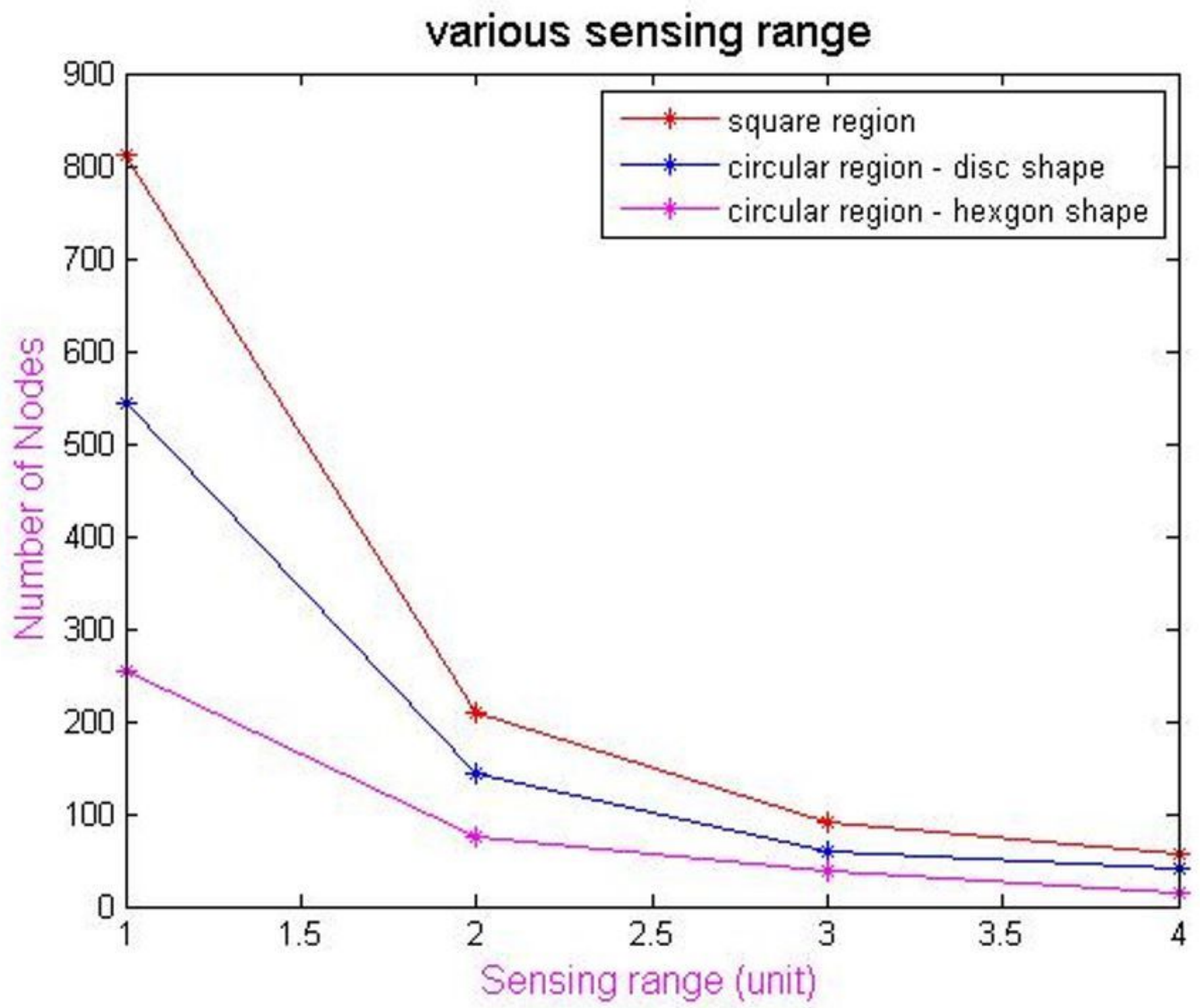

Figure 15

Comparison of required nodes in square and hexagon coverage regions based on various sensing range 